\title{
Regularized Image System for Stokes Flow Outside a Solid Sphere
}

\author{
Jacek K. Wróbel ${ }^{\mathrm{a}, *}$, Ricardo Cortez ${ }^{\mathrm{a}, *}$, Douglas Varela ${ }^{\mathrm{b}}$, Lisa Fauci $^{\mathrm{a}}$ \\ ${ }^{a}$ Department of Mathematics and Center for Computational Science, Tulane University, United States \\ ${ }^{b}$ DVC Academic Consulting, San Pablo, CA, United States
}

\begin{abstract}
The image system for a three-dimensional flow generated by regularized forces outside a solid sphere is formulated and implemented as an extension of the method of regularized Stokeslets. The method is based on replacing a point force given by a delta distribution with a smooth localized function and deriving the exact velocity field produced by the forcing. In order to satisfy zero-flow boundary conditions at a solid sphere, the image system for singular Stokeslets is generalized to give exact cancellation of the regularized flow at the surface of the sphere. The regularized image system contains the same elements as the singular counterpart but with coefficients that depend on a regularization parameter. As this parameter vanishes, the expressions reduce to the image system of the singular Stokeslet. The expression relating force and velocity can be inverted to compute the forces that generate a given velocity boundary condition elsewhere in the flow. We present several examples within the context of biological flows at the microscale in order to validate and highlight the usefulness of the image system in computations.
\end{abstract}

Keywords: regularized Stokeslets, method of images, flow outside a sphere

\section{Introduction}

Biological systems at the microscale often involve flows around spherical objects. Successful fertilization in mammals requires sperm penetration of the viscoelastic layer surrounding the spherical ovum [1]. In the case of invertebrates, it has been shown that encounter rates between a swimming sperm and the egg depend upon the shear force produced by fluid motion near the rotating egg [2]. In addition, many microorganisms have spherical cell bodies. The flow dynamics near the cell body are important for motility [3] as well as nutrient exchange [4]. At these length scales, inertia is negligible, and the three-dimensional fluid motion may be described by the incompressible Stokes equations

$$
\begin{aligned}
& \mu \Delta \mathbf{u}=\nabla p-\mathbf{F}(\mathbf{x}), \\
& \nabla \cdot \mathbf{u}=0
\end{aligned}
$$

where $\mu$ is the fluid viscosity, $\mathbf{u}$ is the fluid velocity, $p$ is the pressure, and $\mathbf{F}$ is the external force per unit volume. When the external force is given by a delta distribution, $\mathbf{F}(\mathbf{x})=\mathbf{f} \delta(\mathbf{x}-\mathbf{y})$, the fundamental solution $\mathbf{u}$ is known as the Stokeslet, which is singular at the point $\mathbf{y}$ where the force is applied.

\footnotetext{
*Please address correspondence to J.K. Wróbel or R. Cortez

Email addresses: jwrobel@tulane.edu (Jacek K. Wróbel), rcortez@tulane.edu (Ricardo Cortez), dvarela@davinci-camp.com (Douglas Varela), fauci@tulane.edu (Lisa Fauci)
} 
The linearity of Stokes flow makes it possible to modify the Stokeslet kernel to analytically enforce zero velocity on certain surfaces. For the case of a solid planar surface, this is accomplished using the method of images, where a Stokeslet and other singular solutions of Stokes equations are placed at the image point: the reflection of $\mathbf{x}$ across the plane. Blake $[5,6]$ used Fourier transforms to derive the images for a (singular) Stokeslet, rotlet, source, and (Stokeslet) doublet. By differentiating with respect to the pole, Pozrikidis [7] derived the image systems for the Stokeslet, source and dipole.

In the case of a solid sphere, the fluid velocity generated by a Stokeslet outside the sphere must satisfy the zero-flow boundary condition at its surface. Oseen [8] in 1927 derived the Green's function (Stokeslet) for this flow based on a velocity ansatz that satisfied the boundary condition and a correction to enforce zero divergence. Nigam and Srinivasan [9] and Higdon [10] discovered that Oseen's formulation could be interpreted as a line distribution of singularities in the interior of the sphere. Specifically, they found that the image system of a radially-directed Stokeslet can be decomposed into a Stokeslet, a potential dipole, and a stresslet with poles at the image point. The image system of a transverse Stokeslet (one with no radial component) can be decomposed into a line distribution of Stokeslets, potential dipoles, and Stokeslet doublets extending from the sphere center to the image point. A comprehensive investigation of these distributions is presented by Kim and Karrila [11] and further discussion can be found in [7, 12]. Examples of the use of this method in computational models of self-propelled microorganisms can be found in $[10,13,14,15]$ with some modifications to deal with the singularities of the external Stokeslets and asymptotically in [16].

\subsection{Regularized Stokeslets}

The method of regularized Stokeslets $[17,18]$ was created as a technique for removing the singularities at the location of the forces. Instead of a point force represented by a delta distribution, the external force in Eq. (1a) is assumed to be given by $\mathbf{F}(\mathbf{x})=\mathbf{f} \phi_{\epsilon}(\mathbf{x}-\mathbf{y})$, where $\phi_{\epsilon}(\mathbf{x})$ is a radially symmetric smooth function concentrated at the origin, similar to a Gaussian. The parameter $\epsilon>0$ controls how concentrated the function is near zero. An exact solution $\mathbf{u}(\mathbf{x})$ of the Stokes equations for this force is called a regularized Stokeslet. For example, the function

$$
\phi_{\epsilon}(\mathbf{x})=\frac{15 \epsilon^{4}}{8 \pi\left(|\mathbf{x}|^{2}+\epsilon^{2}\right)^{7 / 2}}
$$

leads to the regularized Stokeslet in component form

$$
8 \pi \mu u_{j}(\mathbf{x})=S_{j k} f_{k}=\left(\frac{1}{R}+\frac{\epsilon^{2}}{R^{3}}\right) \delta_{j k} f_{k}+\frac{\hat{x}_{j} \hat{x}_{k}}{R^{3}} f_{k}
$$

where $\delta_{j k}$ is the Kronecker delta (Einstein summation notation assumed) and we use the notation $\hat{\mathbf{x}}=\mathbf{x}-\mathbf{y}$ and $R=\sqrt{|\hat{\mathbf{x}}|^{2}+\epsilon^{2}}$. The method of regularized Stokeslets provides a nonsingular velocity field everywhere, including the points where the forces are applied. We mention also that in the limit as $\epsilon \rightarrow 0$, Eq. (3) reduces to the traditional Stokeslet with singularities at $\mathbf{y}$.

The method of images for a planar surface has been adapted to be used in combination with the method of regularized Stokeslets [19, 20]. Applications of the planar regularized images can be found in [21, 22, 23, 24, $25,26,27,28,29]$. On the other hand, thus far, the image system for flows bounded by a sphere applies only 
to singular solutions (Stokeslet) in the flow. We extend this work by deriving the corresponding image system for regularized Stokeslets. The result is an explicit formula relating the regularized forces generating the flow to the fluid velocity that vanishes analytically at the surface of a sphere. The linear dependence of the velocity on the forces can be inverted to solve for the forces that generate a flow with given velocity constraints. Several examples are presented to display the use of the method in applications.

\section{Image system for a sphere using regularized Stokeslets}

Given a sphere of radius $a$ centered at the origin, we assume that a Stokeslet is located at point $\mathbf{y}^{*}$ outside the sphere and define $r_{j}^{*}=x_{j}-y_{j}^{*}, r^{*}=\left|\mathbf{x}-\mathbf{y}^{*}\right|$ and $R^{* 2}=r^{* 2}+\epsilon^{* 2}$. Here, $\mathbf{x}$ is the point where the velocity is evaluated.

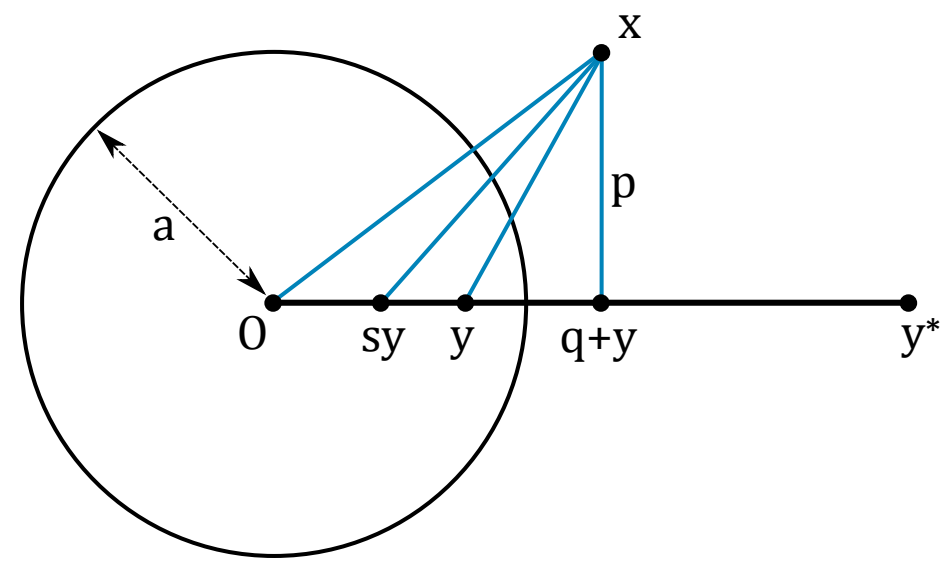

Figure 1: Schematic diagram and notation. The diagram shows the projection of the solid sphere onto a plane defined by the velocity evaluation point $\mathbf{x}$, the Stokeslet location $\mathbf{y}^{*}$ and the center of the sphere.

The image point is defined as $\mathbf{y}$ with

$$
y_{j}=\frac{a^{2}}{\left|\mathbf{y}^{*}\right|^{2}} y_{j}^{*}
$$

and we set $r_{j}=x_{j}-y_{j}$ and $r=|\mathbf{x}-\mathbf{y}|$. We will use the fact that for $|\mathbf{x}|=a$ (at the sphere surface) the distances satisfy $r=\frac{a}{\left|\mathbf{y}^{*}\right|} r^{*}$, which motivates scaling the regularization parameter at the image point by $\epsilon=\epsilon^{*} a /\left|\mathbf{y}^{*}\right|$ so that

$$
R^{2}=r^{2}+\epsilon^{2}=\frac{a^{2}}{\left|\mathbf{y}^{*}\right|^{2}} R^{* 2}
$$

The original Stokeslet. The flow due to a Stokeslet of strength $\vec{f}$ is given by $8 \pi \mu u_{j}=S_{j k} f_{k}$, where

$$
S_{j k}=\left(\frac{1}{R^{*}}+\frac{\epsilon^{* 2}}{R^{* 3}}\right) \delta_{j k}+r_{j}^{*} r_{k}^{*} \frac{1}{R^{* 3}} .
$$

To translate this to the image point, we use the expansion $r_{j}^{*}=r_{j}+y_{j} h$ where $h=1-a^{2} /|\mathbf{y}|^{2}$, so that

$$
S_{j k}=\frac{|\mathbf{y}|}{a}\left(\frac{1}{R}+\frac{\epsilon^{2}}{R^{3}}\right) \delta_{j k}+\frac{|\mathbf{y}|^{3}}{a^{3}} \frac{1}{R^{3}}\left(r_{j} r_{k}+h\left(r_{j} y_{k}+r_{k} y_{j}\right)+h^{2} y_{j} y_{k}\right) .
$$


The regularized elements required at the image point are Stokeslets, doublets, dipoles and rotlets:

$$
\begin{aligned}
S_{j k} & =\left(\frac{1}{R}+\frac{\epsilon^{2}}{R^{3}}\right) \delta_{j k}+r_{j} r_{k} \frac{1}{R^{3}} \\
\Delta_{j k l} & =-\frac{3 \epsilon^{2}}{R^{5}} \delta_{j k} r_{l}+\left(-\delta_{j k} r_{l}+\delta_{j l} r_{k}+\delta_{k l} r_{j}\right) \frac{1}{R^{3}}-3 r_{j} r_{k} r_{l} \frac{1}{R^{5}} \\
D_{j k} & =\left(-\frac{1}{R^{3}}+\frac{3 \epsilon^{2}}{R^{5}}\right) \delta_{j k}+3 r_{j} r_{k} \frac{1}{R^{5}} \\
\mathcal{R}_{j k} & =\epsilon_{j k l} r_{l} \frac{1}{R^{3}} .
\end{aligned}
$$

Here, the index notation $\epsilon_{j k l} v_{k} b_{l}$ represents the $j$-th component of $\mathbf{v} \times \mathbf{b}$. Because of the spherical symmetry, a general force $\vec{f}$ in three dimensions located at $\mathbf{y}^{*}$ can be decomposed into a radial component and a transverse component. Let $e_{k}=y_{k}^{*} /\left|\mathbf{y}^{*}\right|$ be the radial unit vector and define the transverse unit vector $e^{\perp}$ through the decomposition $f_{k}=\left(f_{j} e_{j}\right) e_{k}+\left[f_{k}-\left(f_{j} e_{j}\right) e_{k}\right]=f^{n} e_{k}+f^{\tau} e_{k}^{\perp}$. In the following derivation we adapt the work of Kim and collaborators $[11,12]$ to the case of regularized Stokeslets.

\subsection{Radial forces}

We assume first that $f_{k}=f y_{k} /|\mathbf{y}|=f e_{k}$, so the original Stokeslet in Eq. (4) can be written as

$$
f e_{k} S_{j k}=f w\left(\frac{1}{R}+\frac{\epsilon^{2}}{R^{3}}\right) e_{j}+f w^{3} \frac{1}{R^{3}}\left(r_{j} q+h|\mathbf{y}|\left(r_{j}+q e_{j}\right)+h^{2}|\mathbf{y}|^{2} e_{j}\right)
$$

where $q=r_{k} e_{k}$ and $w=|\mathbf{y}| / a$.

In this case we will use only the three following image elements: a radial Stokeslet of strength $g e_{k}$, a doublet of strength $\tilde{\sigma} e_{k} e_{l}$ (i.e. a stresslet, since the doublet strength is a symmetric tensor), and a radial dipole of strength $\tilde{d} e_{k}$. Using their respective expressions from Eq. (5)-(7) we have that

$$
\begin{aligned}
g e_{k} S_{j k} & =g\left(\frac{1}{R}+\frac{\epsilon^{2}}{R^{3}}\right) e_{j}+g q r_{j} \frac{1}{R^{3}} \\
\tilde{\sigma} e_{k} e_{l} \Delta_{j k l} & =\tilde{\sigma}\left(-\frac{3 \epsilon^{2}}{R^{5}} e_{j} q+r_{j} \frac{1}{R^{3}}-3 r_{j} q^{2} \frac{1}{R^{5}}\right) \\
\tilde{d} e_{k} D_{j k} & =\tilde{d}\left(\left(-\frac{1}{R^{3}}+\frac{3 \epsilon^{2}}{R^{5}}\right) e_{j}+3 q r_{j} \frac{1}{R^{5}}\right) .
\end{aligned}
$$

Based on the singular case [11], we expect that $g=\frac{1}{2} f w^{3}-\frac{3}{2} f w$. Using this value and adding all components gives a velocity

$$
\begin{aligned}
8 \pi \mu u_{j} & =f e_{k} S_{j k}+g e_{k} S_{j k}+\tilde{\sigma} e_{k} e_{l} \Delta_{j k l}+\tilde{d} e_{k} D_{j k} \\
& =e_{j}\left[f w^{3} h|\mathbf{y}|(q+h|\mathbf{y}|) \frac{1}{R^{3}}-\frac{1}{2} f w\left(1-w^{2}\right)\left(\frac{1}{R}+\frac{\epsilon^{2}}{R^{3}}\right)-\tilde{\sigma} \frac{3 \epsilon^{2}}{R^{5}} q+\tilde{d}\left(-\frac{1}{R^{3}}+\frac{3 \epsilon^{2}}{R^{5}}\right)\right] \\
& +r_{j}\left[\left(f w^{3} h|\mathbf{y}|-\frac{3}{2} f w\left(1-w^{2}\right) q+\tilde{\sigma}\right) \frac{1}{R^{3}}-3 \tilde{\sigma} q^{2} \frac{1}{R^{5}}+3 \tilde{d} q \frac{1}{R^{5}}\right] .
\end{aligned}
$$

Since the vector $\mathbf{r}=\mathbf{x}-\mathbf{y}$ will have a radial component and a component perpendicular to $\mathbf{y}$, we write $r_{j}=q e_{j}+p e_{j}^{\perp}$. For notational convenience, we define $\alpha$ and $\beta$ through the formula

$$
q=r_{j} e_{j}=\frac{a^{2}-|\mathbf{y}|^{2}}{2|\mathbf{y}|}-\frac{r^{2}}{2|\mathbf{y}|}=\frac{1}{2}\left(\frac{\epsilon^{2}}{|\mathbf{y}|}-h|\mathbf{y}|-\frac{R^{2}}{|\mathbf{y}|}\right)=\alpha-\beta R^{2}
$$


Using these two expressions, the velocity at the sphere surface must satisfy

$$
\begin{aligned}
0 & =e_{j}\left[R \frac{3 \beta}{2}\left(f w^{3}-f w+2 \tilde{\sigma} \beta\right)\right. \\
& +\frac{1}{R}\left[\left(-2 f w^{3} h|\mathbf{y}| \beta+\frac{1}{2} f w^{3}-\frac{1}{2} f w\right)-2 \alpha \beta\left(\frac{3}{2} f w^{3}-\frac{3}{2} f w+3 \tilde{\sigma} \beta\right)-\beta \tilde{\sigma}+3 \beta^{2}(\tilde{d}-\tilde{\sigma} \alpha)\right] \\
& +\frac{1}{R^{3}}\left[\epsilon^{2} \frac{1}{2}\left(f w^{3}-f w+6 \tilde{\sigma} \beta\right)+f w^{3}(\alpha+h|\mathbf{y}|)^{2}+\alpha^{2}\left(\frac{1}{2} f w^{3}-\frac{3}{2} f w+3 \tilde{\sigma} \beta\right)-(6 \alpha \beta+1)(\tilde{d}-\tilde{\sigma} \alpha)\right] \\
& \left.+\frac{3}{R^{5}}\left(\epsilon^{2}+\alpha^{2}\right)(\tilde{d}-\tilde{\sigma} \alpha)\right] \\
& +p e_{j}^{\perp}\left[-\frac{1}{R} \frac{3 \beta}{2}\left(f w^{3}-f w+2 \tilde{\sigma} \beta\right)+\frac{1}{R^{3}}\left(\frac{3}{2} \alpha f w\left(w^{2}-1\right)+f w^{3} h|\mathbf{y}|+\tilde{\sigma}+6 \tilde{\sigma} \alpha \beta-3 \tilde{d} \beta\right)+\frac{3}{R^{5}} \alpha(\tilde{d}-\tilde{\sigma} \alpha)\right]
\end{aligned}
$$

\subsubsection{Cancelation in the directions of $\mathbf{y}$}

The velocity component in the $e_{j}$ direction must vanish for all $R$, so that looking at the coefficients of $R$ and $R^{-5}$, we set

$$
f w^{3}-f w+2 \tilde{\sigma} \beta=0, \quad \tilde{d}-\tilde{\sigma} \alpha=0
$$

which leads to

$$
0=u_{j} e_{j}=\frac{1}{R}(-2 \beta)\left[\operatorname{faw}^{2}\left(w^{2}-1\right)+\tilde{\sigma}\right]
$$

where we have used $w=|\mathbf{y}| / a$ to write $w^{2} h=w^{2}-1, w h|\mathbf{y}|=a\left(w^{2}-1\right)$ and the definitions $\alpha=\frac{1}{2}\left(\epsilon^{2} /|\mathbf{y}|-h|\mathbf{y}|\right)$ and $\beta=1 / 2|\mathbf{y}|$. Note that these conditions make the coefficients of $R^{-1}$ and $R^{-3}$ vanish also. We conclude that

$$
\begin{aligned}
g & =\frac{1}{2} f w\left(w^{2}-3\right) \\
\tilde{\sigma} & =f a w^{2}\left(1-w^{2}\right) \\
\tilde{d} & =\frac{1}{2} f w\left(1-w^{2}\right)\left[a^{2}\left(1-w^{2}\right)+\epsilon^{2}\right] .
\end{aligned}
$$

It can be verified that with these choices the velocity in the perpendicular direction $u_{j} e_{j}^{\perp}$ also vanishes.

In summary, a radial Stokeslet outside the sphere at $\mathbf{y}^{*}$ has images at $\mathbf{y}$ consisting of the elements in Eq. (10)-(12) with coefficients given in Eq. (13).

\subsubsection{Transverse forces}

In this section we assume $\vec{f}$ is transversal, so that $f_{k}=f e_{k}^{\perp}$ and the original Stokeslet is

$$
f e_{k}^{\perp} S_{j k}=f w\left(\frac{1}{R}+\frac{\epsilon^{2}}{R^{3}}\right) e_{j}^{\perp}+f w^{3} \frac{1}{R^{3}}\left(r_{j} p+h|\mathbf{y}| p e_{j}\right)
$$

where $p=r_{j} e_{j}^{\perp}$ and $w=|\mathbf{y}| / a$. Since $\vec{r}=\mathbf{x}-\mathbf{y}$ can have any direction, we write $r_{j}=x_{j}-y_{j}=q e_{j}+p e_{j}^{\perp}+t e_{j}^{\perp \perp}$, where $q=r_{j} e_{j}, t=r_{j} e_{j}^{\perp \perp}$ and $e^{\perp \perp}=e \times e^{\perp}$. Note that $r^{2}=p^{2}+q^{2}+t^{2}$.

Following the analysis in [11], in the transverse case we will make use of all four image elements below plus a distribution of Stokeslets, dipoles and rotlets along the line segment connecting the center of the sphere and the image point. We begin with the elements at $\mathbf{y}$, which are: a Stokeslet of strength $g e_{k}^{\perp}$, a doublet of strength 
$\sigma e_{k}^{\perp} e_{l}$, a dipole of strength $d e_{k}^{\perp}$ and a rotlet of strength $\lambda\left(\epsilon_{k m n} e_{m}^{\perp} e_{n}\right)$.

$$
\begin{aligned}
g e_{k}^{\perp} S_{j k} & =g\left(\frac{1}{R}+\frac{\epsilon^{2}}{R^{3}}\right) e_{j}^{\perp}+g p r_{j} \frac{1}{R^{3}} \\
\sigma e_{k}^{\perp} e_{l} \Delta_{j k l} & =\sigma\left(-\frac{3 \epsilon^{2}}{R^{5}} e_{j}^{\perp} q+\left(-e_{j}^{\perp} q+e_{j} p\right) \frac{1}{R^{3}}-3 r_{j} p q \frac{1}{R^{5}}\right) \\
d e_{k}^{\perp} D_{j k} & =d\left(-\frac{1}{R^{3}}+\frac{3 \epsilon^{2}}{R^{5}}\right) e_{j}^{\perp}+3 d p r_{j} \frac{1}{R^{5}} \\
\lambda\left(\epsilon_{k m n} e_{m} e_{n}^{\perp}\right) \mathcal{R}_{j k} & =\lambda\left(\epsilon_{k m n} e_{m} e_{n}^{\perp}\right) \epsilon_{j k l} r_{l} \frac{1}{R^{3}}=\lambda\left(-e_{j} p+e_{j}^{\perp} q\right) \frac{1}{R^{3}}
\end{aligned}
$$

As before, we expect $g=\frac{1}{2} f w^{3}-\frac{3}{2} f w$ so we add the 5 elements and use the decomposition of $r_{j}$ to get the velocity contribution of these elements to be

$$
\begin{aligned}
& e_{j}^{\perp}\left[\frac{1}{2} f w\left(w^{2}-1\right)\left(\frac{1}{R}+\frac{\epsilon^{2}}{R^{3}}\right)+\left(\frac{3 p^{2}}{2} f w\left(w^{2}-1\right)-\sigma q-d+\lambda q\right) \frac{1}{R^{3}}+3(d-q \sigma)\left(p^{2}+\epsilon^{2}\right) \frac{1}{R^{5}}\right] \\
+ & p e_{j}\left[\left(f w^{3} h|\mathbf{y}|+\sigma-\lambda+q \frac{3}{2} f w\left(w^{2}-1\right)\right) \frac{1}{R^{3}}+3 q(d-q \sigma) \frac{1}{R^{5}}\right] \\
+ & p t e_{j}^{\perp \perp}\left[\frac{3}{2} f w\left(w^{2}-1\right) \frac{1}{R^{3}}+3(d-q \sigma) \frac{1}{R^{5}}\right]
\end{aligned}
$$

where the unknown scalars are $\sigma, d$ and $\lambda$.

Line integrals of 3 elements. In addition to the elements above, we will need three more elements that are obtained from the integral along the line segment from the center of the sphere to $\mathbf{y}$ of a Stokeslet, a doublet and a dipole. These are proportional to a scalar $\rho$ which must be determined. Let $s \in[0,1]$ and note that $x_{j}-s y_{j}=r_{j}+(1-s)|\mathbf{y}| e_{j}=p e_{j}^{\perp}+t e_{j}^{\perp \perp}+(q+(1-s) \mathbf{y}) e_{j}$. We define $X^{2}=|\mathbf{x}-s \mathbf{y}|^{2}=p^{2}+t^{2}+(q+(1-s)|\mathbf{y}|)^{2}$ and $Q^{2}=X^{2}+\epsilon^{2}$.

Then the integral of Stokeslets with strengths $\rho|\mathbf{y}| s e_{k}^{\perp}$ is

$$
I_{S}=\rho|\mathbf{y}|^{2} \int_{0}^{1} e_{j}^{\perp}\left(\frac{1}{Q}+\frac{\epsilon^{2}+p^{2}}{Q^{3}}\right) s+\frac{s p t e_{j}^{\perp \perp}}{Q^{3}}+\frac{s p[q+(1-s)|\mathbf{y}|] e_{j}}{Q^{3}} d s .
$$

Similarly, the integral of dipoles of strengths $\rho|\mathbf{y}|\left(\ell^{2} s-|\mathbf{y}|^{2} s^{3}\right) e_{k}^{\perp}$ is

$$
\begin{aligned}
I_{D} & =\rho|\mathbf{y}|^{2} \int_{0}^{1} e_{j}^{\perp}\left(-\frac{1}{Q^{3}}+\frac{3\left(\epsilon^{2}+p^{2}\right)}{Q^{5}}\right)\left(\ell^{2} s-|\mathbf{y}|^{2} s^{3}\right)+\frac{3 p t e_{j}^{\perp \perp}}{Q^{5}}\left(\ell^{2} s-|\mathbf{y}|^{2} s^{3}\right) \\
& +\frac{3 p[q+(1-s)|\mathbf{y}|] e_{j}}{Q^{5}}\left(\ell^{2} s-|\mathbf{y}|^{2} s^{3}\right) d s
\end{aligned}
$$

where $\ell$ is a length scale to be determined. The integral of rotlets of strengths $2 \rho|\mathbf{y}|^{2} s^{2}\left(\epsilon_{k m l} e_{m} e_{l}^{\perp}\right)$ is

$$
I_{R}=2 \rho|\mathbf{y}|^{3} \int_{0}^{1}\left([q+(1-s)|\mathbf{y}|] e_{j}^{\perp}-p e_{j}\right)\left(\frac{1}{Q^{3}}\right) s^{2} d s .
$$

Using a recursion formula for the integrals (see Appendix A), we arrive at the following expression for the sum $I_{S}+I_{D}+I_{R}$ evaluated at a point $\mathbf{x}$ on the sphere

$$
\begin{aligned}
& \rho e_{j}^{\perp}\left[-\frac{R}{2}+\left(a^{2}+\epsilon^{2}+|\mathbf{y}|^{2}\right) \frac{1}{R}-\frac{1}{2}\left(\left(a^{2}+\epsilon^{2}-|\mathbf{y}|^{2}\right)^{2}+4 t^{2}|\mathbf{y}|^{2}\right) \frac{1}{R^{3}}\right] \\
+ & \rho|\mathbf{y}| p e_{j}\left[-\frac{1}{R}+\left(a^{2}+\epsilon^{2}-|\mathbf{y}|^{2}\right) \frac{1}{R^{3}}\right]+2 \rho p t|\mathbf{y}|^{2} e_{j}^{\perp \perp} \frac{1}{R^{3}}
\end{aligned}
$$


Combining Eq. (22) with Eq. (18) gives the velocity at the sphere surface

$$
\begin{aligned}
& e_{j}^{\perp}\left[\frac{1}{2} f w\left(w^{2}-1\right)\left(\frac{1}{R}+\frac{\epsilon^{2}}{R^{3}}\right)+\left(\frac{3 p^{2}}{2} f w\left(w^{2}-1\right)-\sigma q-d+\lambda q\right) \frac{1}{R^{3}}+3(d-q \sigma) p^{2} \frac{1}{R^{5}}\right. \\
+ & \left.3(d-\sigma q) \frac{\epsilon^{2}}{R^{5}}+\rho\left(-\frac{R}{2}+\left(a^{2}+\epsilon^{2}+|\mathbf{y}|^{2}\right) \frac{1}{R}-\frac{1}{2}\left(\left(a^{2}+\epsilon^{2}-|\mathbf{y}|^{2}\right)^{2}+4 t^{2}|\mathbf{y}|^{2}\right) \frac{1}{R^{3}}\right)\right] \\
+ & p e_{j}\left[\left(f w^{3} h|\mathbf{y}|+\sigma-\lambda+q \frac{3}{2} f w\left(w^{2}-1\right)\right) \frac{1}{R^{3}}+3 q(d-q \sigma) \frac{1}{R^{5}}+\rho|\mathbf{y}|\left(-\frac{1}{R}+\left(a^{2}+\epsilon^{2}-|\mathbf{y}|^{2}\right) \frac{1}{R^{3}}\right)\right] \\
+ & p t e_{j}^{\perp \perp}\left[\frac{3}{2} f w\left(w^{2}-1\right) \frac{1}{R^{3}}+3(d-q \sigma) \frac{1}{R^{5}}+2 \rho|\mathbf{y}|^{2} \frac{1}{R^{3}}\right]
\end{aligned}
$$

which vanishes with the choices

$$
\begin{aligned}
& g=\frac{1}{2} f w\left(w^{2}-3\right) \\
& \sigma=-a f w^{2}\left(1-w^{2}\right) \frac{a^{2}\left(1-w^{2}\right)-\epsilon^{2}}{a^{2}\left(1-w^{2}\right)+\epsilon^{2}} \\
& d=-\frac{1}{2} f w\left(1-w^{2}\right)\left(a^{2}\left(1-w^{2}\right)-\epsilon^{2}\right) \\
& \lambda=-\frac{2 a^{3} f w^{2}\left(1-w^{2}\right)^{2}}{a^{2}\left(1-w^{2}\right)+\epsilon^{2}} \\
& \rho=\frac{3 f\left(1-w^{2}\right)^{2}}{2 w\left(a^{2}\left(1-w^{2}\right)+\epsilon^{2}\right)}
\end{aligned}
$$

where $w=|\mathbf{y}| / a$. These expressions provide exact cancellation of the velocity field at the surface of the sphere for any nonnegative value of $\epsilon$. The limit as $\epsilon \rightarrow 0$ gives the same images in [11] for the singular Stokeslet.

In summary, a transverse Stokeslet outside the sphere at $\mathbf{y}^{*}$ has images at $\mathbf{y}$ consisting of the elements in Eq. (14)-(17) plus the integrals in Eq. (19)-(21) with coefficients given in Eq. (23). In practice the integrals are computed analytically and computed as a single element at the image point.

\section{Numerical Examples}

We present several numerical examples, motivated by biological flows at the microscale, that show the utility of the image system.

\subsection{Example 1: Flow due to Three Forces}

In the first example, we consider three random forces in the $z=0$ plane and compute the flow they generate in the $z=0$ plane. Figure $2 \mathrm{~b}$ shows the forces and velocity field computed with the image system derived in Section 2 around a sphere of radius 0.8. The region occupied by the sphere has been shaded for visualization. Since the forces are isolated and there is no particular discretization length scale, the regularization parameter was set arbitrarily to $\epsilon=0.01$. For comparison we have also included in Figure 2a the flow produced by the forces in the absence of the sphere. Without the image system, the surface of the sphere would have to be discretized with regularized forces that enforce the zero flow condition at the surface. This would require a potentially costly solution of a large linear system. 

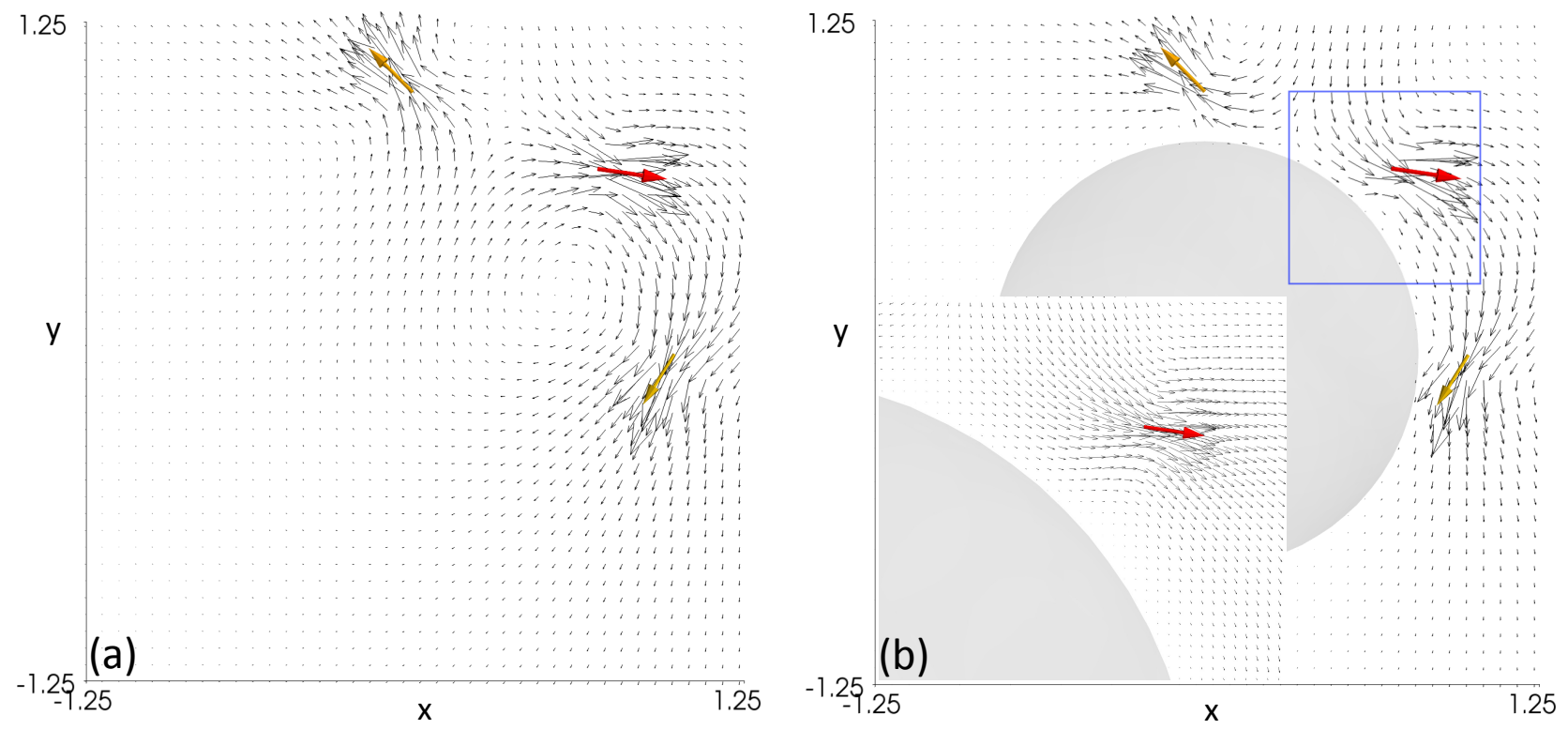

Figure 2: (a, b) Veloctiy fields at the $z=0$ plane due to three random point forces evaluated utilizing free space regularized Stokeslet and solid sphere regularized Stokeslet, respectively. The sphere of radius $r_{s}=0.8$, point forces at distance 0.2 from the surface of the sphere at points $(1,0,0),(\sqrt{2} / 2, \sqrt{2} / 2,0)$ and $(0,1,0)$, the regularization parameter $\epsilon=0.01$.

\subsection{Example 2: Viscoelastic Network in Stokes Flow}

In biological systems, viscous fluids often contain polymers that provide elastic properties. Such viscoelastic flows play important roles in transport by cilia in mucociliary transport in the lungs [30], and sperm penetration of the oocyte cumulus complex in mammalian fertilization [31], for example. Motivated by the latter, we consider a network of elastic links surrounding a sphere and the deformation of the network due to an applied force in the fluid. The sphere is assumed to be stationary, and the fluid velocity must be zero at its surface. The network consists of a mesh of thickness 0.5 (five layers of 1002 nodes each) around a sphere of radius 0.9. Each node in the mesh is connected to its 26 nearest neighbors by links consisting of a spring-dashpot combination depicted in Figure 3. The dashpot allows for the resting length of the spring to change so that the network configuration after applying a force may not be equal to the initial configuration. More details can be found in [32].

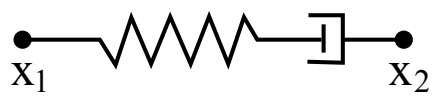

Figure 3: Schematic of a spring-dashpot link.

In our example, a single regularized force at a distance of 1.6 above the sphere is applied for 0.5 time units. The fluid motion generated by the force deforms the network, which in turn, produces forces at the nodes. The forces at the nodes $\mathbf{x}_{1}$ and $\mathbf{x}_{2}$ due to a single link connecting them are

$$
\mathbf{f}_{s}\left(\mathbf{x}_{1}\right)=-\mathbf{f}_{s}\left(\mathbf{x}_{2}\right)=\ell_{0}^{2} E\left(\frac{\left\|\mathbf{x}_{2}-\mathbf{x}_{1}\right\|}{\ell(t)}-1\right) \frac{\mathbf{x}_{2}-\mathbf{x}_{1}}{\left\|\mathbf{x}_{2}-\mathbf{x}_{1}\right\|},
$$


where $\ell(t)$ denotes the element resting length and $E$ is the stiffness constant and $\ell_{0}=\ell(0)$ an initial resting length. The spring resting lengths change dynamically with the equation

$$
\frac{d \ell(t)}{d t}=\frac{E \ell_{0}}{\eta}\left(\frac{\left\|\mathbf{x}_{2}-\mathbf{x}_{1}\right\|}{\ell(t)}-1\right)
$$

where $\eta$ is a constant. The total force at each network node is computed by summing over the individual links emanating from that node [32]. Figure 4 shows the network at the times when the force is initially applied and when it is removed, which is also the time of maximum deformation of the network.
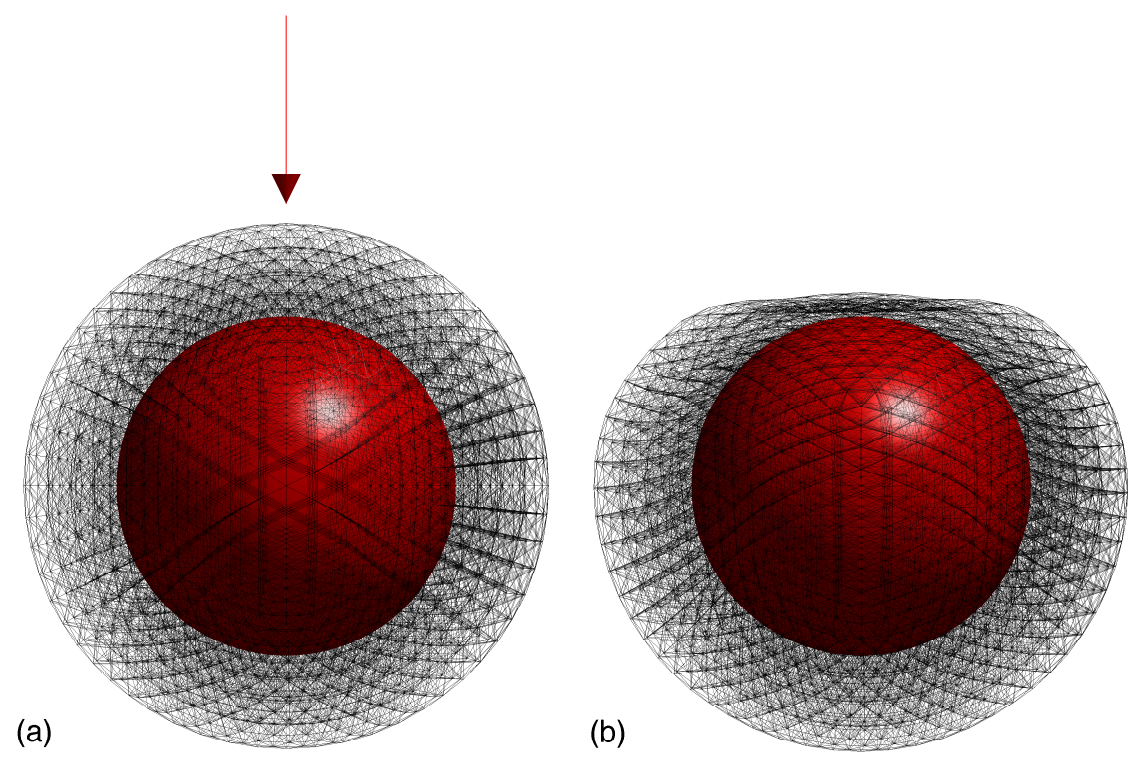

Figure 4: Viscoelatic network attached to a solid sphere subject to radial point force (a) at time $t_{0}=0$ and (b) at time $t_{1}=0.5(\eta=5)$. Sphere radius $r_{s}=0.9$, network composed of five equidistant, crosslinked layers of 1002 nodes each, the network's thickness $L \approx 0.5$, the applied force magnitude is 300 located at a distance 1.6 from the surface of the sphere.

Given the location of the nodes at any time, the forces at the nodes are computed using Eq. (24)-(25) and the velocity of the nodes is then computed using the image system by direct evaluation. The network nodes are updated using the fluid velocity at their location. The computational advantage is that the sphere appears only virtually and its surface does not have to be discretized in any way. In fact, if the image system was not used, zero velocity conditions would have to be imposed on the nodes of the discretized surface. This would give rise to a linear system to solve for the forces on those nodes so that the no-slip condition is satisfied. This system would be of size $3 M \times 3 M$, where $M$ is the total number of nodes on the sphere. We emphasize that, with images, no such linear system needs to be solved.

\subsection{Example 3: Helical swimmer}

In the previous example of the deformation of a viscoelastic mesh around a rigid sphere, forces at the nodes of the mesh were specified, and the mesh evolved with the velocities at those nodes due to those forces. In contrast, we now examine the swimming of a microorganism whose flagellar dynamics are specified, and the 
forces that result in the specified kinematics must be solved for. This model microorganism has a spherical cell body of radius $A$ and a helical flagellum of length $L$ connected to it at one point. We assume the organism's shape relative to itself (i.e., up to translation and rotation) is given by the curve

$$
\mathbf{X}(s, t)=(s, E(s) \alpha \cos (k s-\gamma t), E(s) \alpha \sin (k s-\gamma t))
$$

where $E(s)=1-e^{-k_{e}^{2} s^{2}}$ so that the helical wave amplitude is damped in a short region near the cell body. The function has property $E(0)=0, E^{\prime}(0)=0$ and grows rapidly to value one so that the helix has mostly constant amplitude $\alpha$ but there is a single point of attachment to the body for all times (see Figure 5a). This setup was considered by Higdon in [15], where slender body theory for the flagellum was coupled to the drag on the sphere to compute the overall swimming speed and power consumption of the model organism.

(a)

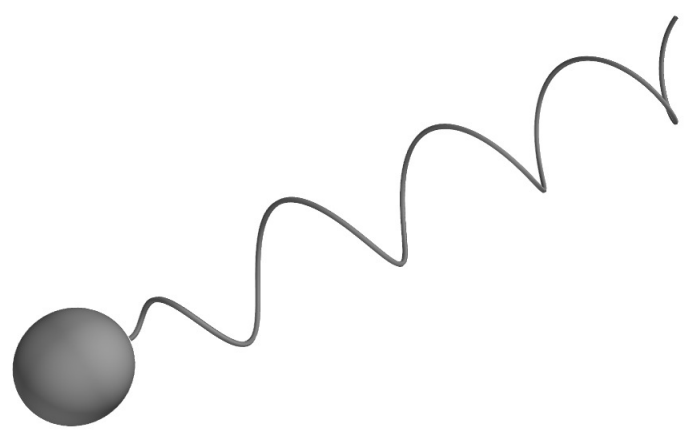

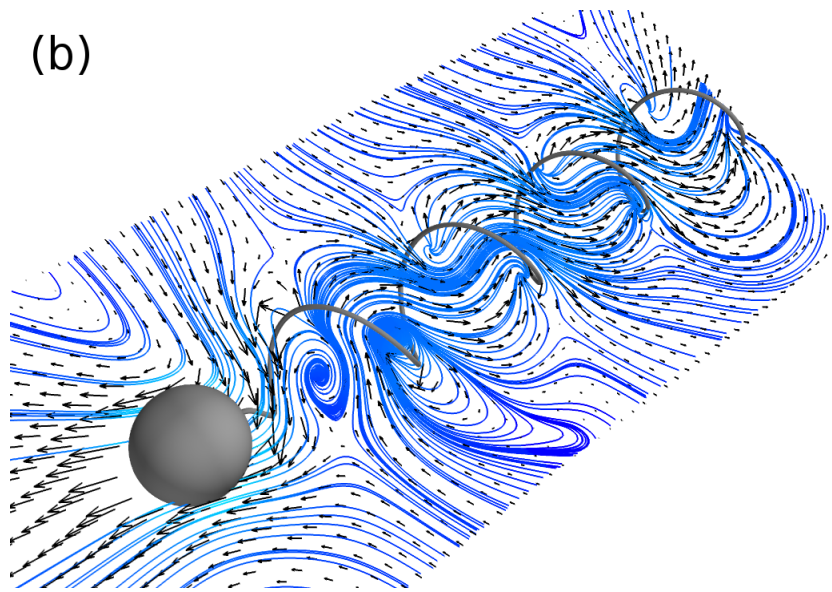

Figure 5: (a) Schematic helical swimmer and (b) fluid flow pattern (velocity field and streamlines) on its section plane.

The flagellum's velocity is prescribed by differentiating Eq. (26) with respect to $t$

$$
\mathbf{u}_{d}(s, t)=\frac{\partial \mathbf{X}(s, t)}{\partial t}
$$

Here, $\mathbf{u}_{d}(s, t)$ is a "deformation velocity" that the flagellum would have in the absence of the fluid. Once it interacts with the fluid, the organism (cell body and flagellum) will also experience translation and rotation. To account for this rigid body motion we place a Stokeslet-dipole combination of strength $\mathbf{f}_{0}$ and a rotlet of strength $\mathbf{L}$ at the center of the sphere. Therefore, if $\mathbf{x}_{c}$ represents the center of the sphere at time $t$ and for $0 \leq s_{0} \leq L$, the total velocity at $\mathbf{X}\left(s_{0}, t\right)$ is given by

$$
\mathbf{u}_{d}\left(s_{0}, t\right)+\mathbf{U}+\boldsymbol{\Omega} \times\left(\mathbf{x}-\mathbf{x}_{c}\right)=\frac{1}{\mu} \int_{0}^{L} \mathbf{G}\left(\mathbf{X}\left(s_{0}, t\right), \mathbf{X}(s, t)\right) \mathbf{f}(s) d s+\mathbf{U}_{\text {trans }}\left(\mathbf{X}\left(s_{0}, t\right)\right)+\mathbf{U}_{\text {rot }}\left(\mathbf{X}\left(s_{0}, t\right)\right)
$$

where $\mathbf{G}$ is the image Stokeslet kernel. Here, $\mathbf{U}_{\text {trans }}(\mathbf{x})$ is the velocity due to the Stokeslet-dipole combination at the center of the sphere, where the unknown strength $\mathbf{f}_{0}$ is related to the rigid body translation by $\mathbf{f}_{0}=$ $6 \pi \sqrt{A^{2}+\epsilon^{2}} \mathbf{U}$ (see Eq. (B.1)-(B.2) in Appendix B for the full expressions). $\mathbf{U}_{\text {rot }}(\mathbf{x})$ is the velocity at $\mathbf{x}$ due to the rotlet at the center of the sphere whose strength $\mathbf{L}$ is related to the rigid body rotation by $\mathbf{L}=4 \pi\left(A^{2}+\epsilon^{2}\right)^{3 / 2} \boldsymbol{\Omega}$ (see Eq. (B.3)-(B.4) in Appendix B for details). 
In computations, we discretize the flagellum with $N$ points equally spaced in arc length so that the point separation is $\Delta s=L /(N-1)$. After approximating the integral, Eq. (28) becomes a system of $3 N$ equations for the $3 N+6$ unknowns $\mathbf{U}, \Omega$ and $\mathbf{f}_{n}(n=1, \ldots, N)$. The final 6 equations come from enforcing zero net force and zero net torque of the organism since there is no external force acting on the system. Care must be taken to ensure that all forces, including those at the image points inside the sphere, are accounted for. Given a force $\mathbf{f}$ outside the sphere, we decompose it into its radial and transverse components $\mathbf{f}=\mathbf{f}^{\|}+\mathbf{f}^{\perp}$. The force and torque due to the images of the radial component are

$$
\mathbf{f}^{I \|}=g \mathbf{f}^{\|} / f \quad \text { and } \quad \mathbf{M}^{\|}=0 .
$$

The force due to the images of the transverse component is

$$
g+\int_{0}^{1} \rho|\mathbf{y}|^{2} \ell d \ell=g+\frac{\rho}{2}|\mathbf{y}|^{2}=g+\frac{\rho}{2} a^{2} w^{2}
$$

and the torque due to the transverse component of the Stokeslets at the images is

$$
\mathbf{y} \times g e^{\perp}+\int_{0}^{1}(\ell \mathbf{y}) \times\left(\rho|\mathbf{y}| \ell e^{\perp}\right)|\mathbf{y}| d \ell=\mathbf{y}^{*} \times\left(g w^{2}\right) e^{\perp}+\mathbf{y}^{*} \times\left(\frac{1}{3} \rho a^{2} w^{4}\right) e^{\perp} .
$$

Similarly we compute contributions to the torque from all image elements and arrive at

$$
\mathbf{f}^{I \perp}=\left(g+\frac{\rho}{2} a^{2} w^{2}\right) e^{\perp} \quad \text { and } \quad \mathbf{M}^{\perp}=\mathbf{y}^{*} \times \mathbf{T}, \quad \text { where } \quad \mathbf{T}=\left(g w^{2}+\rho a^{2} w^{4}+\frac{w(\lambda-\sigma)}{a}\right) e^{\perp} .
$$

We point out that in the limit of vanishing regularization, the quantities in Eq. (29)-(30) reduce to $\mathbf{f}^{I}=$ $\frac{w}{2}\left(w^{2}-3\right) \mathbf{f}^{\|}+\left(-\frac{3}{4} w-\frac{1}{4} w^{3}\right) \mathbf{f}^{\perp}$ and $\mathbf{M}^{\perp}=-w^{3} e^{\perp}$ shown by Higdon in [14], where $w=|\mathbf{y}| / A=A /\left|\mathbf{y}^{*}\right|$. Similar models were proposed by Higdon in $[10,15]$.

Thus the zero net force and zero net torque conditions to be enforced are

$$
\mathbf{f}_{0}+\sum_{n=1}^{N}\left(\mathbf{f}_{n}+\mathbf{f}_{n}^{I}\right) \Delta s=0 \quad \text { and } \quad 2 \mathbf{L}+\sum_{n=1}^{N}\left(\mathbf{X}_{n}-\mathbf{x}_{c}\right) \times\left(\mathbf{f}_{n}+\mathbf{T}_{n}\right) \Delta s=0
$$

where $\mathbf{f}_{0}$ is the force at the sphere center that produces the translation velocity $\mathbf{U}$ and $2 \mathbf{L}$ is the torque produced by the rotlet at the center of the sphere which generates the angular velocity $\boldsymbol{\Omega}$ (see Appendix B). Once the linear system has been solved, the velocity at any point $\mathbf{x}$ in the fluid is given by

$$
\mathbf{u}(\mathbf{x})=\frac{1}{\mu} \sum_{n=1}^{N} \mathbf{G}\left(\mathbf{x}, \mathbf{X}_{n}\right) \mathbf{f}_{n} \Delta s+\mathbf{U}_{\text {trans }}(\mathbf{x})+\mathbf{U}_{\text {rot }}(\mathbf{x}) .
$$

Comment: While the computation of the Stokeslet flow with sphere images has been derived based on the radial and transversal decomposition of each force, when solving the inverse problem, the forces have to be written with respect to a common coordinate system. This is accomplished with a change of basis. For example, the radial component of a force $\mathbf{f}$ located at $\mathbf{y}^{*}$ is $\mathbf{f}^{\|}=\mathbf{e} \mathbf{e}^{T} \mathbf{f}$, where $\mathbf{e}=\mathbf{y}^{*} /\left|\mathbf{y}^{*}\right|$ and the superscript $T$ indicates the transpose. Then the velocity due to the radial force satisfies

$$
\mu \mathbf{u}(\mathbf{x})=\mathbf{G}^{r a d}\left(\mathbf{x}, \mathbf{y}^{*}\right) \mathbf{f}^{\|}=\mathbf{G}^{\operatorname{rad}}\left(\mathbf{x}, \mathbf{y}^{*}\right)\left(\mathbf{e e}^{T}\right) \mathbf{f}
$$


which amounts to modifying the image Stokeslet kernel by the appropriate transformation. Similarly, the transverse component $\left(I-\mathbf{e e}^{T}\right) \mathbf{f}$ is decomposed into two directions, $\mathbf{e}_{1}$ and $\mathbf{e}_{2}$, that are orthogonal to each other and to the radial direction. Then the velocity due to the transverse force satisfies

$$
\mu \mathbf{u}(\mathbf{x})=\mathbf{G}^{\text {trans }}\left(\mathbf{x}, \mathbf{y}^{*}\right) \mathbf{e}_{1} \mathbf{e}_{1}^{T}\left(I-\mathbf{e e}^{T}\right) \mathbf{f}+\mathbf{G}^{\text {trans }}\left(\mathbf{x}, \mathbf{y}^{*}\right) \mathbf{e}_{2} \mathbf{e}_{2}^{T}\left(I-\mathbf{e e}^{T}\right) \mathbf{f} .
$$

As in [15] we record the average swimming speed and the power consumption for different configurations of the swimmer by changing the parameter $L / A$. The average swimming speed normalized by the wave speed $V=\gamma / k$ is expressed as

$$
\bar{U} / V=\frac{k\left(\mathbf{U} \cdot \boldsymbol{\Omega}_{p}\right)}{\gamma\left|\boldsymbol{\Omega}_{p}\right|} .
$$

where $\boldsymbol{\Omega}_{p}=\left(\Omega_{1}-\gamma, \Omega_{2}, \Omega_{3}\right)$. The dimensionless power consumption is

$$
\eta_{0}^{-1}=\bar{P} / 6 \pi \mu A \bar{U}^{2}
$$

where the power is expressed as

$$
\bar{P}=\sum_{n=1}^{N} \mathbf{f}\left(s_{n}\right) \cdot \mathbf{u}_{d}\left(s_{n}\right) \Delta s .
$$

Figure $6 \mathrm{a}$ shows the average swimming speed and Figure $6 \mathrm{~b}$ shows the power consumption as functions of the number of wavelengths, $N_{\lambda}$. The results show close agreement with same quantities reported in Figures 3-4 of [15], where the flagellum was approximated using slender-body theory in order to address the singularity of the Stokeslet. The regularization parameter in our simulations was set to $\epsilon=0.02 \mathrm{~A}$ so that it equals the radius of the slender body used in [15]. Convergence studies for a fixed regularization parameter, but refining the discretization along the flagellum, were performed (not shown here), and demonstrate that velocities and power computations with 500 flagellar nodes differed by less than one percent from those computed using 8000 flagellar nodes. Figures $6 \mathrm{a}, \mathrm{b}$ also show the average swimming speed and power consumption at $L / A=5$ computed not with images, but with a full discretization of the spherical cell body. These values are indistinguishable from those computed using the regularized image system. For each $N_{\lambda}$ the flagellum was discretized with 500 nodes, and the sphere surface was discretized with 5292 nodes. While a $1500 \times 1500$ linear system needed to be solved for node forces when the images for the sphere were used, a $17376 \times 17376$ linear system needed to be solved when images were not used. The zero velocity boundary condition had to be imposed one each sphere node. In each case, the linear system was solved using the Python built-in solver based upon GESV. Sweeping through all of the values of $N_{\lambda}$ in Figures $6 \mathrm{a}$,b took about 17 seconds on a single processor desktop when images were used compared to about 20 minutes when the sphere was discretized. In fact, this was a coarse discretization of the sphere, because the distances between nodes on the sphere surface were considerably larger than the distances between nodes along the flagellum.

\subsection{Example 4: Dinoflagellates Model}

Dinoflagellates are unicellular phytoplankton that swim due to the action of two flagella. A longitudinal flagellum propagates approximately planar wave and a transverse helical flagellum that is wrapped around the cell and propagates helical waves; see Figure 7a. The cell body of the microorganism is nearly spherical and has 

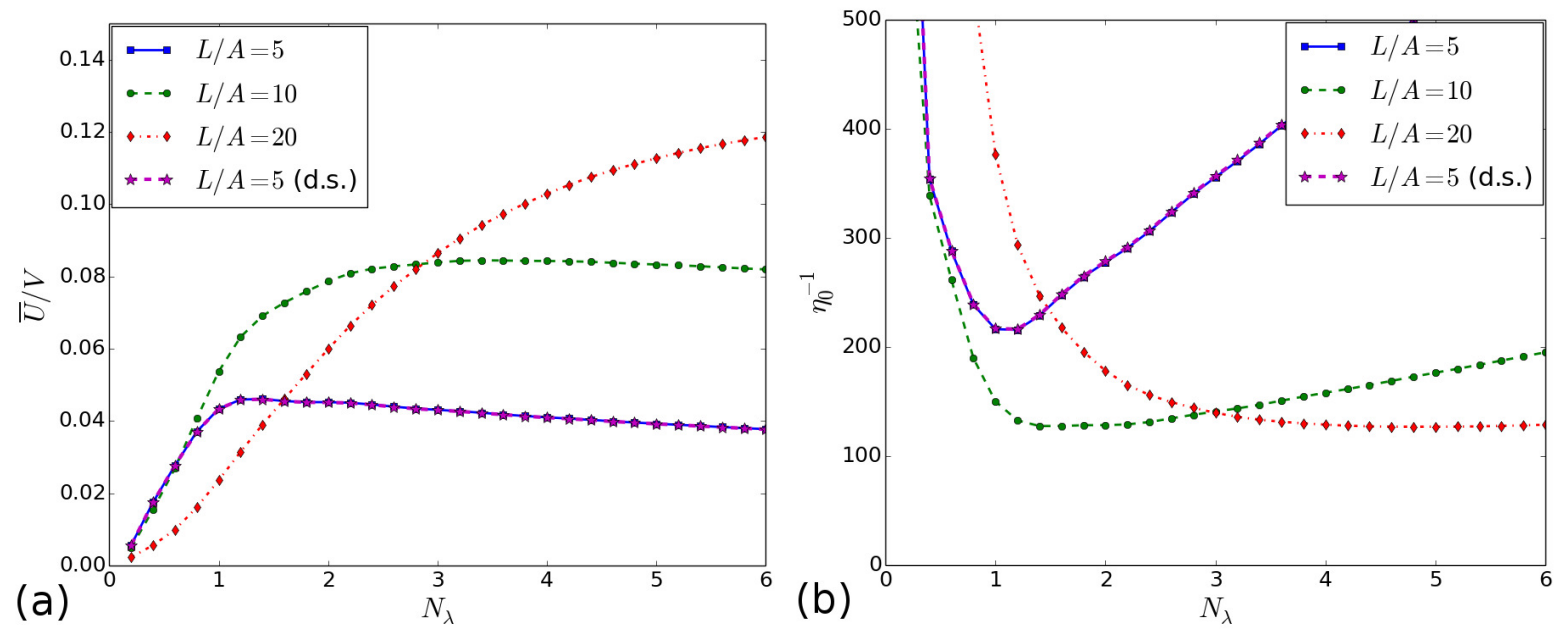

Figure 6: (a) Average swimming speed and (b) power consumption in the non-dimensional form (34) as functions of the number of waves, respectively. The curves are shown for three different length flagella and $\epsilon / A=0.02$ discretized at $N=L / \epsilon$ points. The wave parameters used were $\alpha k=1$ and $k / k_{e}=1$. Also shown are the computed values when a full discretization of the sphere is used $(L / A=5$ d.s. $)$

been observed to rotate and translate about its longitudinal axis, producing helical swimming trajectories [33, $34]$.

(a)
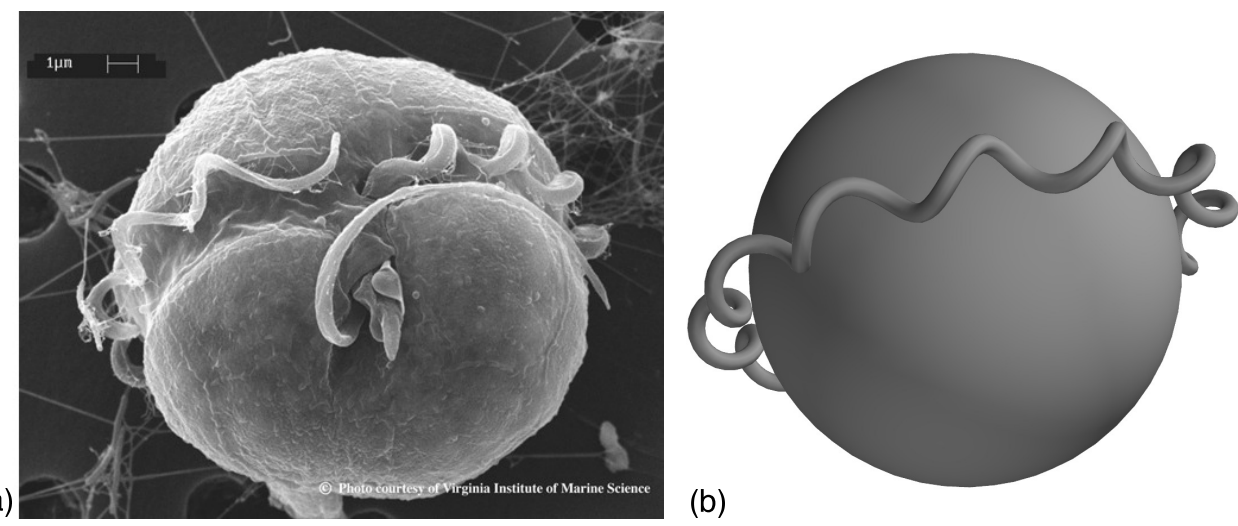

(b)

Figure 7: (a) The dinoflagelate Pfiesteria piscicida (Copyright 2011, Virginia Institute of Marine Science (VIMS)/College of William and Mary.) (b) Model proposed in [35].

An idealized model of the transverse flagellum as a closed waving ring, in the absence of any cell body, demonstrated that both rotational and translational motion of the ring could result from the propagation of a helical wave around its length in a viscous fluid [36]. Here we focus on the fluid motion generated by the transverse flagellum, which is represented by a closed helical tube around the equator of a sphere. The fluid motion is generated by a wave propagating along the helical tube; see Figure 7b. We idealize the model swimmer by not including an attachment of the flagellum to the spherical cell body. Moreover, in contrast with the helical swimmer example in the previous section, here we choose to represent the flagellum not by a curve, but by a tube with surface forces. This surface distribution of regularized Stokeslets, while more costly than the 
centerline distribution, has been shown to be more accurate for helices with appreciable curvature or thickness [37]. In [35], we investigated the motility of this model dinoflagellate using regularized Stokeslets without the use of sphere images, but a full discretization of the sphere.

The centerline of the closed helical tube is given parametrically by the equation

$$
\mathbf{X}_{c}(s, t)=\left\{\begin{array}{l}
X(s, t)=\left[r_{0}-R \sin \left(n_{p} s / r_{0}-\gamma t\right)\right] \cos \left(s / r_{0}\right) \\
Y(s, t)=\left[r_{0}-R \sin \left(n_{p} s / r_{0}-\gamma t\right)\right] \sin \left(s / r_{0}\right) \\
Z(s, t)=R \cos \left(n_{p} s / r_{0}-\gamma t\right)
\end{array}\right.
$$

where $0 \leq s \leq 2 \pi r_{0}$. The helical centerline is wrapped around a circle of radius $r_{0}$ and has amplitude $R$ and wavelength $\lambda=2 \pi r_{0} / n_{p}$, where $n_{p}$ denotes the number of pitches. As time $t$ progresses the toroidal spiral travels around the torus with speed $\omega_{s}=\gamma \lambda / 2 \pi$ and period $T=2 \pi / \gamma$.

A tube of radius $r_{h}$ around $\mathbf{X}_{c}(s, t)$ is generated by defining a local set of orthonormal vectors $(\mathbf{T}(s, t), \mathbf{N}(s, t), \mathbf{B}(s, t))$, where $\mathbf{T}(s, t)$ is tangent to the centerline. Then the tube surface is

$$
\mathbf{X}_{h}(s, \theta, t)=\mathbf{X}_{c}(s, t)+r_{h}(\mathbf{N}(s, t) \sin \theta+\mathbf{B}(s, t) \cos \theta)
$$

where $0 \leq \theta \leq 2 \pi$. The local coordinates used here are

$$
\mathbf{T}(s, t)=\frac{\mathbf{X}_{c}^{\prime}(s, t)}{\left\|\mathbf{X}_{c}^{\prime}(s, t)\right\|}, \quad \mathbf{N}(s, t)=\frac{\mathbf{T}(s, t) \times \mathbf{X}_{c}^{\prime \prime}(s, t)}{\left\|\mathbf{X}_{c}^{\prime \prime}(s, t)\right\|} \quad \text { and } \quad \mathbf{B}(s, t)=\mathbf{T}(s, t) \times \mathbf{N}(s, t),
$$

where the prime indicates differentiation with respect to $s$. The prescribed tube velocity is then given by differentiating Eq. (37) with respect to time

$$
\dot{\mathbf{X}}_{h}(s, \theta, t)=\dot{\mathbf{X}}_{c}(s, t)+r_{h}(\dot{\mathbf{N}} \sin \theta+\dot{\mathbf{B}} \cos \theta)-r_{h}(\dot{\mathbf{B}} \cdot \mathbf{N})(\mathbf{N} \cos \theta-\mathbf{B} \sin \theta)
$$

where the last term simply removes any tube cross-sectional rotation (twist) due to the choice of local coordinates (see Appendix C).

Using discrete values of the parameters $s$ and $\theta$, we discretize the tube surface at time $t$ with $N$ points $\mathbf{X}_{n}$ and set their velocities to $\mathbf{u}_{d}\left(\mathbf{X}_{n}\right)$ based on Eq. (38). We add a Stokeslet-dipole combination of strength $\mathbf{f}_{0}$ and a rotlet of strength $\mathbf{L}$ at the center of the sphere as before, and solve Eq. (31) together with

$$
\mathbf{u}_{d}\left(\mathbf{X}_{m}\right)+\mathbf{U}+\boldsymbol{\Omega} \times\left(\mathbf{X}_{m}-\mathbf{x}_{c}\right)=\frac{1}{\mu} \sum_{n=1}^{N} \mathbf{G}\left(\mathbf{X}_{m}, \mathbf{X}_{n}\right) \mathbf{f}_{n} \Delta s+\mathbf{U}_{\text {trans }}\left(\mathbf{X}_{m}\right)+\mathbf{U}_{\text {rot }}\left(\mathbf{X}_{m}\right)
$$

for $m=1, \cdots, N$. This gives the forces $\mathbf{f}_{n}$ as well as $\mathbf{U}$ and $\boldsymbol{\Omega}$.

By construction the model results in transitional and angular velocities along the vertical axis, i.e., $\mathbf{U}=$ $\left(0,0, V_{T}\right)$ and $\boldsymbol{\Omega}=\left(0,0, \Omega_{3}\right)$. We define the rotational velocity of the helical tube as $V_{\perp}=r_{0} \Omega_{3}$ and use the data listed in Table 1 which are the ones used in [35]. For comparison, as in [35], we also perform simulations using the free-space regularized Stokeslets (no images) but including the sphere discretized with $N_{s}$ points, so that in this case the total number of forces is the sum of the sphere points and the helical flagellar points.

In Figure 8 we report the computed normalized values of the translational and rotational velocities of the organism. The values using the method of images nearly coincides with the values obtained by discretizing the 
Table 1: Parameters used in the dinoflagellate simulations using the method of images and using a discretized sphere.

\begin{tabular}{|l|c|l|}
\hline $\begin{array}{l}\text { Helical tube } \\
\text { discretization }\end{array}$ & $r_{0}=0.5, R=0.09, r_{h}=0.035$ and $n_{p}=4$ \\
& $119 \times 6$ distinct patches, $\Delta s \approx 0.0325$ \\
\hline Sphere radius & $r_{s}=0,0.0298,0.0596,0.0894,0.1192,0.1490,0.1788$, \\
& $0.2085,0.2383,0.2681,0.2979,0.3277,0.3575$ \\
\hline Sphere discretization & $N_{s}=0,13,50,113,200,313,450$, & With images \\
\hline Regularization parameter & $613,800,1013,1250,1512,1800$ & $N_{s}=0$ \\
\hline Linear system size & $M=0.019$ & $\epsilon=0.019$ \\
\hline & $3987,4548,5187,5898,6684,7548$ & $M=2148$ \\
\hline
\end{tabular}

sphere along with the flagellum for a wide range of sphere radii (at $r_{s} / \lambda=0.4775$ the sphere surface would touch the tube surface). The results indicate that the rotational velocity about the $z$-axis is largest when there is no sphere and decreases as the sphere radius increases while the translation velocity increases with sphere radius. The method of images has the advantage that the linear system to solve for the forces is always the same size regardless of the sphere radius. On the other hand, discretizing the sphere together with the flagellum increases the size of the linear system as shown in Table 1 leading to a longer computational time once the sphere is large enough $\left(r_{s} / \lambda \approx 0.2\right)$ as shown in Figure 9.
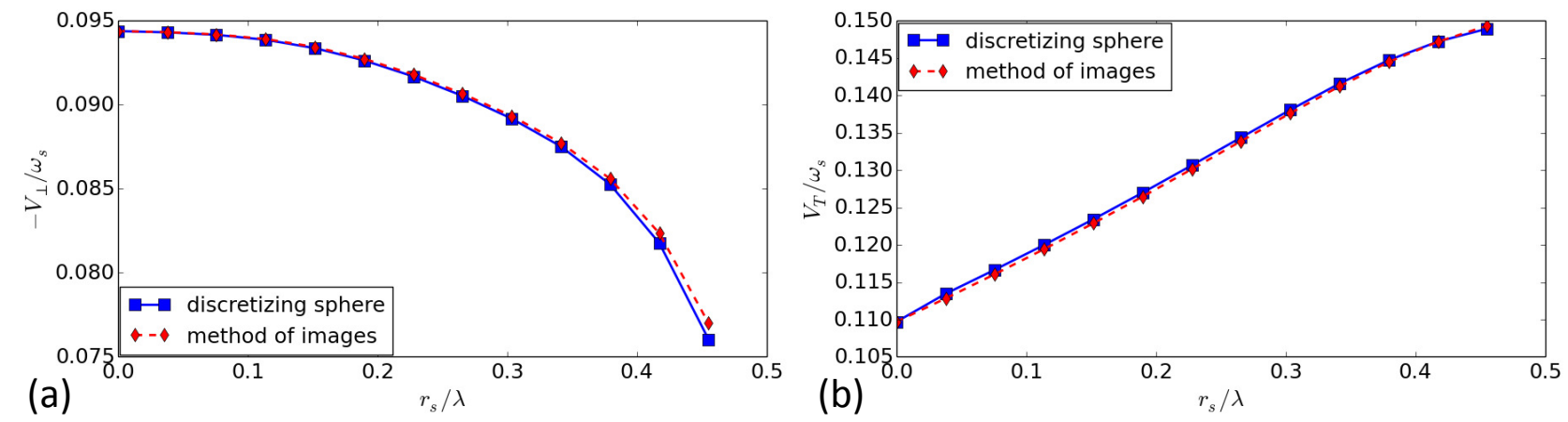

Figure 8: (a) Normalized rotational velocities $\left(V_{\perp} / \omega_{s}\right)$ and (b) normalized translational velocities $\left(V_{T} / \omega_{s}\right)$ both versus normalized spherical radius $\left(r_{s} / \lambda\right)$. The solid curve with square markers corresponds to computations with a discretized sphere surface and using the free-space regularized Stokeslets. The dashed curve with diamond markers corresponds to computations with the sphere images.

Without the computational burden of having to discretize a sphere surface, we compute the flow around a more complex scenario of a helical tube with $n_{p}=13$ pitches as shown in Figure $7 \mathrm{~b}$. In this case we set $\Delta s \approx 0.01$ by discretizing the tube with 572 cross-sections with 12 points each. The geometrical parameters were set to $r_{0}=0.5, r_{h}=0.0175$, and $R=0.045$. Figure 10 shows the flow pattern surrounding the organism for two different sphere sizes, a large one with $r_{s}=r_{0}-R-1.5 r_{h}$ that leaves a gap of $0.5 r_{h}$ between the 


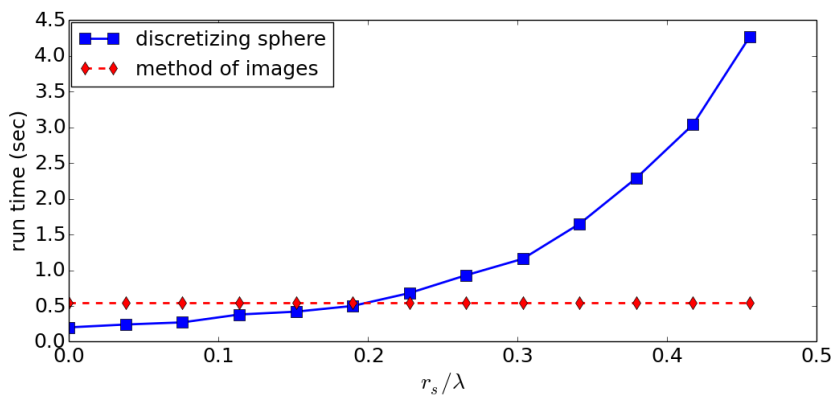

Figure 9: Times for the computation of the dinoflagellate's rotational and translational velocities using the method of images (dashed) and when the sphere surface is discretized (solid). The computations were done on a single processor desktop.

sphere and the tube, and a small sphere with $r_{s}=0.5\left(r_{0}-R-1.5 r_{h}\right)$. The regularization parameter was set to $\epsilon=1.4 \Delta s$. This was based on testing for fluid motion across the tube surface. If $\epsilon$ is too small relative to $\Delta s$, there can be flow across the tube surface between mesh nodes. We chose $\epsilon$ as small as possible so that the flow across the surface is always less than about $2 \%$ of the flagellum speed. This threshold can be reduced with finer discretizations. Figure 10 shows two different perspectives for each case. The helical wave along the closed flagellum generates a ring of rotation around the sphere with a propulsive motion upward. The larger sphere almost plugs the region inside the flagellum, leaving little room for fluid to move between them. The smaller sphere clearly allows more flow between the sphere and the helical tube.

We investigate the effect of the size of the cell body on the velocities by considering two cases: a large sphere and no sphere at all. Figure 11b shows the normalized translational velocities for the cases of no cell body and large cell body $\left(r_{s}=r-R-1.5 r_{h}\right)$ as a function of the number of pitches along the tube. The translational velocity increases with the number of pitches. The comparison clearly shows that for any number of pitches on the tube the organism translates faster with a cell body present. Figure 11a shows the normalized rotational velocity as a function of the number of pitches for the case when there is no cell body and when the cell body is largest. Interestingly, the direction of rotation changes when the number of pitches increases. Zero pitches produces no rotation since it corresponds to a horizontal circular ring. As the number of pitches increases, the rotational speed is negative, giving a rotation that is opposite to that of the propagating wave. The rotational speed then reaches a minimum that depends on the size of the sphere, and begins to grow until it becomes positive once the number of pitches is larger than about 22-24. The magnitude of the rotational velocity is smaller for the case of a large sphere. Spheres of other sizes follow similar trends (not shown).

\subsection{Example 5: Peristaltic pumping}

This example is motivated by physiological systems where rhythmic muscular contractions of a tube cause fluid transport $[38,1,39,40]$. Here we consider a flexible axisymmetric tube of finite length whose cross-sectional radius is given by

$$
r(s, t)=r_{0}+R \sin \left(\frac{2 \pi s}{\lambda}-\gamma t\right),
$$



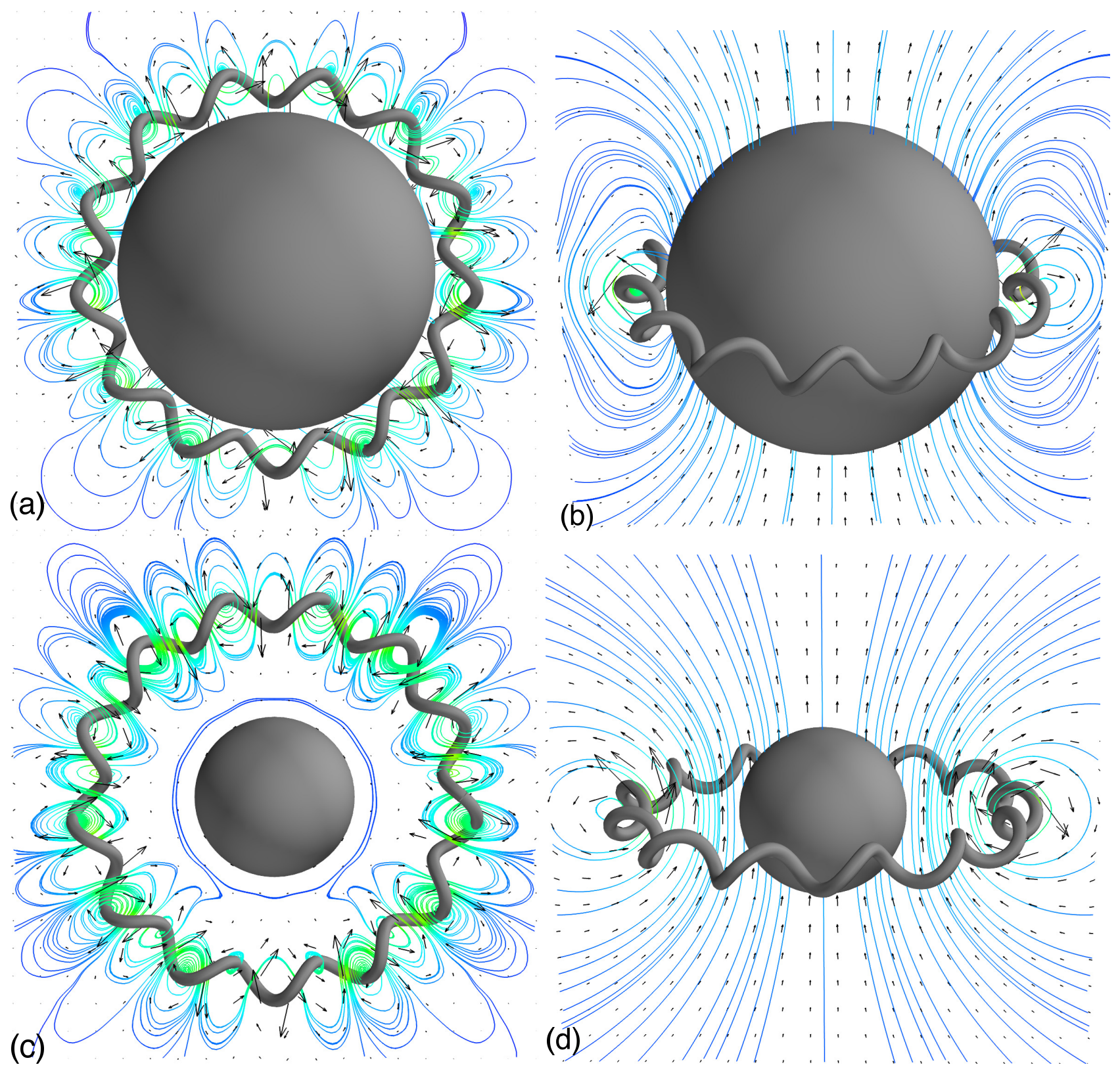

Figure 10: Flow patterns surrounding the organism projected onto horizontal and vertical planes for spheres of radius (a, b) $r_{s}=r_{0}-R-1.5 r_{h}$, and (c, d) $r_{s}=0.5\left(r_{0}-R-1.5 r_{h}\right)$, respectively. The parameters used were $r_{0}=0.5$, tubular radius $r_{h}=0.0175, n_{p}=13$, helix amplitude of $R=0.045$ with $n_{h}=12$ points at each of $n_{c}=572$ cross-sections. The traveling wave frequency was $\gamma=2 \pi$. 

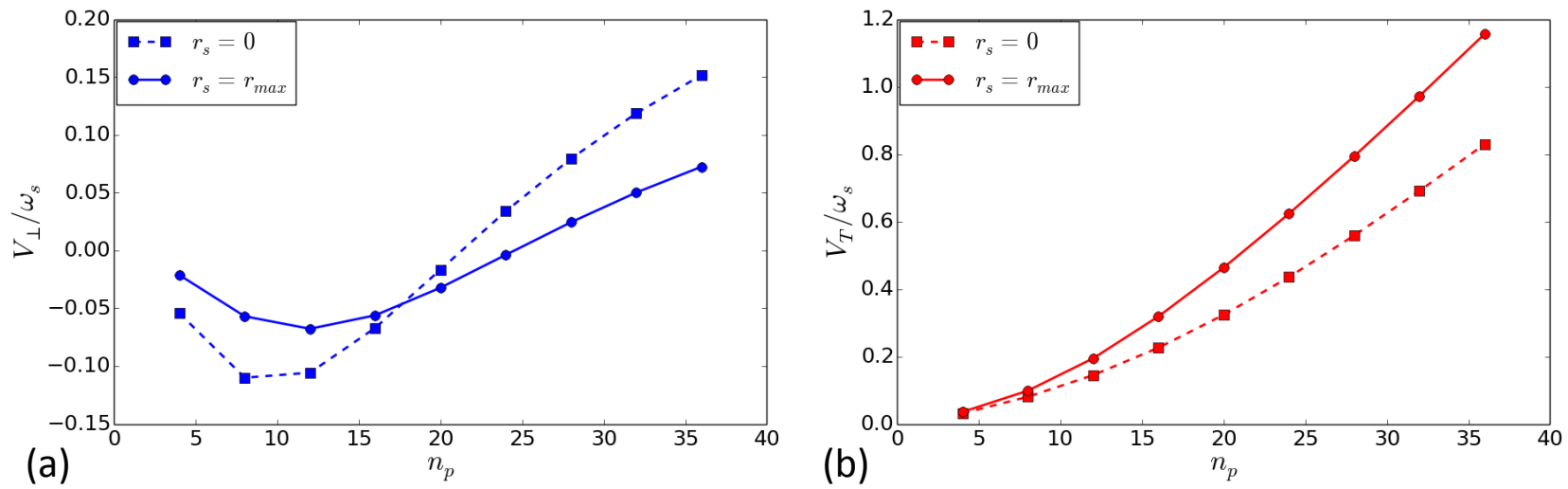

Figure 11: Plots of (a) normalized rotational velocity $V_{\perp} / \omega_{s}$, (b) normalized translational velocity $V_{T} / \omega_{s}$ vs. the number of pitches $n_{p}$, for no cell body $\left(r_{s}=0\right)$ and the maximum cell body size $\left(r_{\max }=r-R-1.5 r_{h}\right)$, respectively.

where $r_{0}$ is the unperturbed tube radius, $R$ is the peristaltic wave amplitude, $\lambda$ is the wavelength, and $\gamma$ is the temporal frequency. That leads to the parametric surface representation

$$
\mathbf{X}(s, \theta, t)=\left\{\begin{array}{l}
X(s, \theta, t)=s \\
Y(s, \theta, t)=r(s, t) \cos \theta \\
Z(s, \theta, t)=r(s, t) \sin \theta
\end{array}\right.
$$

where $0 \leq s \leq L$ and $0 \leq \theta \leq 2 \pi$. As in the previous example, the tube wall has prescribed velocity given by $\mathbf{u}_{d}(s, \theta, t)=\partial \mathbf{X}(s, \theta, t) / \partial t$.

As time evolves, the peristaltic wave, which moves from left to right when $\gamma>0$, generates a net flow inside the tube. However, obstructions in the channel will perturb this flow so we consider a stationary sphere inside the tube and compute the flow using the image system for the sphere. In this example we discretize the tube wall with $N$ points where the unknown forces are located. We find the forces by imposing the velocity at the wall and solving the linear system

$$
\mathbf{u}_{d}\left(\mathbf{X}_{m}, t\right)=\frac{1}{\mu} \sum_{n=1}^{N} \mathbf{G}\left(\mathbf{X}_{m}, \mathbf{X}_{n}\right) \mathbf{f}_{n} r_{n} \Delta s \Delta \theta
$$

for $1 \leq m \leq N$, where $\mathbf{f}_{n}$ and $r_{n}$ are the force and the tube radius at $\mathbf{X}_{n}$. Once the forces are determined, we use the right side of Eq. (42) with $\mathbf{x}$ in place of $\mathbf{X}_{m}$ to compute the fluid velocity at any point $\mathbf{x}$.

We choose dimensionless tube parameters and wave kinematics in Table 2. The surface of the tube is discretized using a $109 \times 80$ mesh, which leads to an approximately uniform mesh spacing of $\Delta s \approx 0.01$. The regularization parameter was set to $\epsilon=1.5 \Delta s$ based on testing for fluid motion across the tube wall as before. Figure 12a shows the geometry of this tube at $t=0.25$, the velocity field and streamlines generated on a plane through the tube axis when the tube is free of obstacles. The flow is characterized by regions of large radius where the flow is from left to right and smaller regions where the tube is narrow and the flow is from right to left. The velocity field shows rotations in the $r x$-plane generated by sections of the tube wall narrowing down while other cross-sections expand. Figures 12b-d show the flow within the tube in the presence of a fixed 
Table 2: Peristaltic tube parameters.

\begin{tabular}{lll}
\hline Parameter & Symbol & Value \\
\hline Tube length & $L=2 \lambda$ & $\pi / 3$ \\
Wavelength & $\lambda$ & $\pi / 6$ \\
Tube radius & $r_{0}$ & 0.125 \\
Amplitude & $R$ & 0.025 \\
Frequency & $\gamma$ & $2 \pi$ \\
Sphere radius & $a$ & 0.05 \\
Sphere center & $\left(x_{c}, y_{c}, z_{c}\right)$ & $(L / 2,-0.045,0)$ \\
\hline
\end{tabular}

spherical obstacle. The sphere is always placed halfway between the inlet and the outlet and 0.045 units off the tube axis. The figures show the disturbance in the flow created by the stationary sphere, including the loss of axial symmetry. The tube is at the same phase in Figures 12a-b. Figure 12b shows the sphere at the narrowest part of the tube and disrupting the flow in nearly half the channel. The velocity field between the sphere and the tube wall must transition quickly from zero at the sphere surface to the prescribed value at the wall. Figures $12 \mathrm{c}-\mathrm{d}$ show the cases corresponding to $t=0.5$ and $t=0.75$, respectively. The vector fields have been scaled the same way in all figures to appreciate changes in velocity magnitude due to the presence of the sphere. In particular, Figure 12d suggests that the peristalsis produces a smaller (left-to-right) volume flow rate at a cross-section through the sphere center compared to the wide section of the tube in Figure 12a. This also affects the velocity field in the narrow sections of the tube.

The use of regularized images eliminates the need to discretize the surface of the spherical object. If we did discretize the sphere so that surface nodes, like tube nodes, were separated by $\Delta s \approx 0.01$, this would have resulted in approximately 314 additional nodes. Here the computational savings achieved using the images is more modest than the previous two examples, with a reduction in size of the linear system from $27102 \times 27102$ down to $26160 \times 26160$.

\section{Discussion and conclusion}

The system of images for regularized Stokeslets for flows outside a solid sphere has been derived. Given a regularized Stokeslet in the fluid domain, the images cancel the flow analytically at the surface of a sphere for any value of the regularization parameter $\epsilon$. This extends previous results on singular spherical images since those expressions can be obtained from the regularized images in the limit $\epsilon \rightarrow 0$. The strength of the regularized image system formulation is in the simulation of flows generated by point forces or forces distributed along filaments in three dimensions since those cases are too singular without some type of regularization. The derivation follows an approach similar to that in [11] and contains the same image elements (Stokeslets, doublets, dipoles and rotlets) inside the sphere although the coefficients are generally dependent on $\epsilon$.

Five examples of applications with different features were presented. The first one is a simple validation 

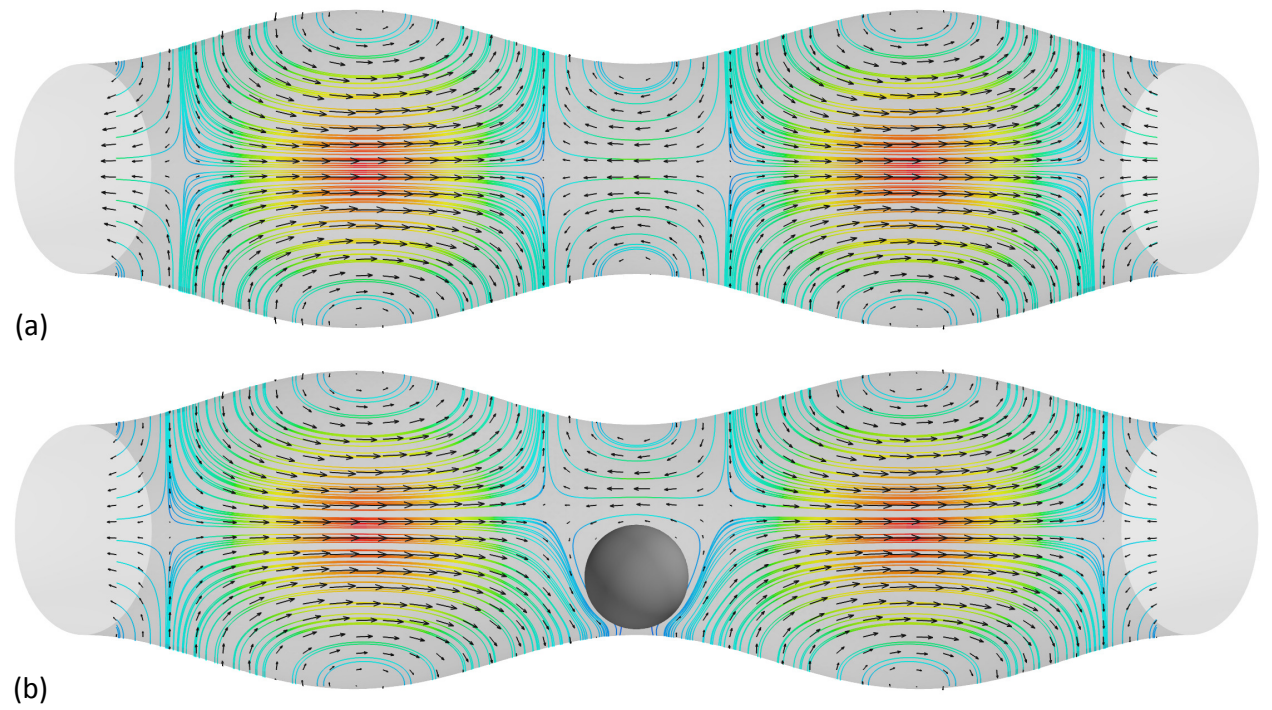

(b)
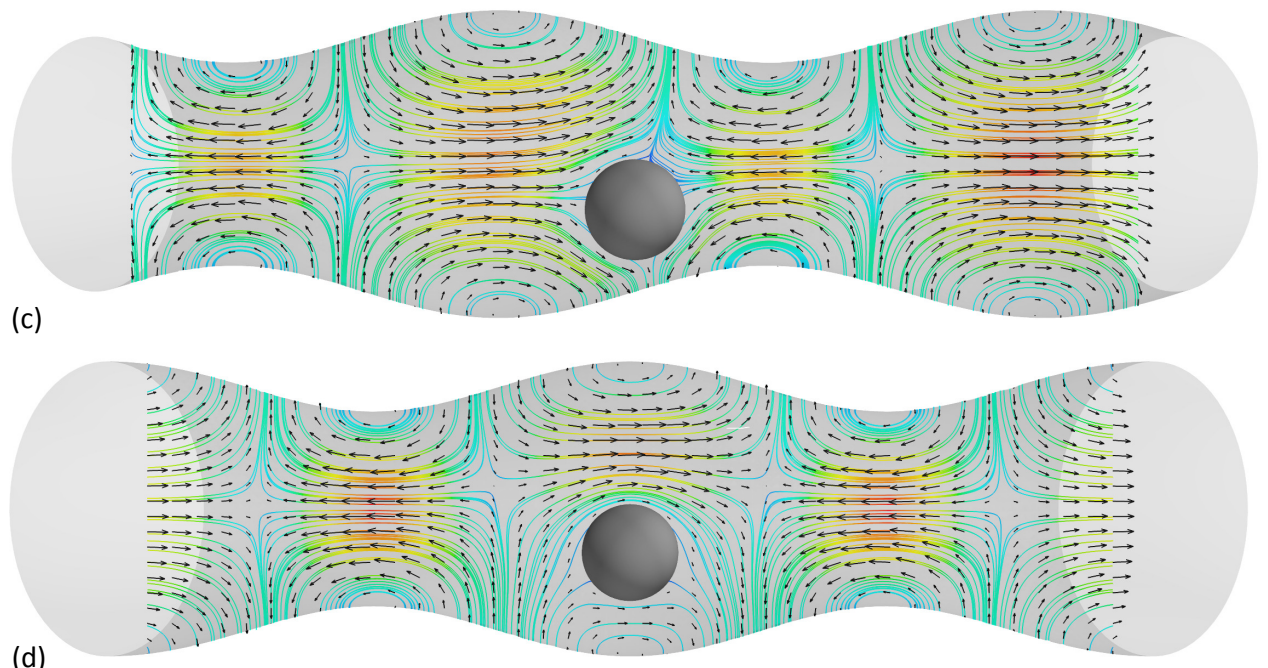

Figure 12: Velocity field and streamlines inside a peristaltic tube with parameters shown in Table 2 (a) without obstacle at time $t=0.25$, (b) $-(\mathbf{d})$ around a spherical particle of radius $a=0.05$ with the center located off the longitudinal axis of the tube by 0.045 and phases $t=0.25, t=0.5$ and $t=0.75$, respectively.

example that shows the flow generated by three regularized point forces near a sphere and a comparison of the flow generated by the same forces but in the absence of the sphere. The second example includes thousands of forces that arise from an elastic network of springs surrounding a sphere. The network deforms due to an external force and all forces become active as the network links (springs with dashpots) deform. In these first two examples, forces are specified and velocities at nodes or other spatial locations may be directly computed using the regularized image system. In contrast, if images were not used and the sphere was discretized with nodes, a linear system would need to be solved to find the forces at the sphere nodes that would result in zero velocities at these nodes, greatly increasing the computational cost of the problem. The following two examples were models of self-propelled microorganisms. A sphere with an attached helical flagellum may represent a bacterium that moves by rotating the flagellum like a corkscrew. In this case, the flagellum velocity relative to 
a fixed sphere is prescribed; however, the entire organism can also experience rigid-body motion. The flagellum forces and the rigid-body motion are computed by enforcing zero net force and zero net torque. Since the flow is smooth everywhere and the velocity is related linearly with the force, the image Stokeslet expression can be inverted to determine forces that would produce prescribed flows at some locations. Once the forces are found, the fluid motion around it can be computed directly. We used this example as validation since that motion was studied extensively by Higdon [14, 15]. Our results for average swimming speed and power consumption compare well with those reported by Higdon. The dinoflagellate example is similar, however, the geometry is different and the flagellum is represented by a tube instead of a curve. The advantage of using images is clear in Figure 9, which compares the computational time to the alternative method of discretizing the sphere surface. The image system is always the same size and only requires changing the parameter that represents the sphere radius. Discretizing the sphere becomes more expensive beyond a certain radius. The final example shows a peristaltic tube with a fixed spherical obstruction inside. We expect that a modification of the linear system for the forces can be used to track a moving spherical particle in the tube. This is left as future work.

\section{Acknowledgments}

This work was funded in part by National Science Foundation grants DMS-1043626 and DMS-1217223.

\section{Appendix A. Simplification of integrals}

We consider

$$
K_{m, n}=\int_{0}^{1} \frac{s^{n}}{Q^{m}} d s, \quad J_{m, n}=\int_{0}^{1} \frac{q+(1-s)|\mathbf{y}|}{Q^{m}} s^{n} d s
$$

where $Q(s)^{2}=p^{2}+t^{2}+\epsilon^{2}+(q+(1-s)|\mathbf{y}|)^{2}$. Define $R^{2}=p^{2}+t^{2}+\epsilon^{2}+q^{2}$. Then one can derive the following identities (for $n \geq 1$ and $m \geq 3$ )

$$
\begin{aligned}
J_{m, 0} & =\frac{1}{|\mathbf{y}|(m-2)}\left[\frac{1}{R^{m-2}}-\frac{1}{\left(R^{2}+(q+|\mathbf{y}|)^{2}-q^{2}\right)^{m / 2-1}}\right] \\
K_{m, 1} & =\frac{-1}{|\mathbf{y}|^{2}(m-2)}\left[\frac{1}{R^{m-2}}-\frac{1}{\left(R^{2}+(q+|\mathbf{y}|)^{2}-q^{2}\right)^{m / 2-1}}\right]+\frac{q+|\mathbf{y}|}{|\mathbf{y}|} K_{m, 0} \\
J_{m, n} & =-\frac{1}{|\mathbf{y}|}\left[\frac{n}{(m-2)} K_{m-2, n-1}-\frac{1}{(m-2)} \frac{1}{R^{m-2}}\right] \\
K_{m, n} & =-\frac{1}{|\mathbf{y}|} J_{m, n-1}+\frac{q+|\mathbf{y}|}{|\mathbf{y}|} K_{m, n-1} \\
K_{m, n+1} & =\frac{1}{|\mathbf{y}|^{2}}\left(\frac{n}{m-2}\right) K_{m-2, n-1}-\frac{1}{|\mathbf{y}|^{2}} \frac{1}{(m-2)} \frac{1}{R^{m-2}}+\frac{q+|\mathbf{y}|}{|\mathbf{y}|} K_{m, n}
\end{aligned}
$$

Using $J_{m, n}$ and $K_{m, n}$ we can write

$$
\begin{gathered}
I_{S}=\rho|\mathbf{y}|^{2} e_{j}^{\perp}\left(K_{1,1}+\left(p^{2}+\epsilon^{2}\right) K_{3,1}\right)+\rho|\mathbf{y}|^{2} p e_{j} J_{3,1}+\rho|\mathbf{y}|^{2} p t e_{j}^{\perp \perp} K_{3,1} \\
I_{D}=\rho|\mathbf{y}|^{2} e_{j}^{\perp}\left(-\ell^{2} K_{3,1}+3 \ell^{2}\left(p^{2}+\epsilon^{2}\right) K_{5,1}+|\mathbf{y}|^{2} K_{3,3}-3|\mathbf{y}|^{2}\left(p^{2}+\epsilon^{2}\right) K_{5,3}\right) \\
+3 \rho|\mathbf{y}|^{2} p e_{j}\left(\ell^{2} J_{5,1}-|\mathbf{y}|^{2} J_{5,3}\right)+3 \rho|\mathbf{y}|^{2} p t e_{j}^{\perp \perp}\left(\ell^{2} K_{5,1}-|\mathbf{y}|^{2} K_{5,3}\right)
\end{gathered}
$$




$$
I_{R}=2 \rho|\mathbf{y}|^{3} e_{j}^{\perp} J_{3,2}-2 \rho|\mathbf{y}|^{3} p e_{j} K_{3,2}
$$

Substituting the values of $J_{m, n}$ below

$$
\begin{gathered}
J_{3,1}=-\frac{1}{|\mathbf{y}|}\left[K_{1,0}-\frac{1}{R}\right] \\
J_{5,1}=-\frac{1}{|\mathbf{y}|}\left[\frac{1}{3} K_{3,0}-\frac{1}{3} \frac{1}{R^{3}}\right] \\
J_{5,3}=-\frac{1}{|\mathbf{y}|}\left[K_{3,2}-\frac{1}{3} \frac{1}{R^{3}}\right] \\
J_{3,2}=-\frac{1}{|\mathbf{y}|}\left[2 K_{1,1}-\frac{1}{R}\right]
\end{gathered}
$$

gives

$$
\begin{gathered}
I_{S}=\rho|\mathbf{y}|^{2} e_{j}^{\perp}\left(K_{1,1}+\left(p^{2}+\epsilon^{2}\right) K_{3,1}\right)-\rho|\mathbf{y}| p e_{j}\left[K_{1,0}-\frac{1}{R}\right]+\rho|\mathbf{y}|^{2} p t e_{j}^{\perp \perp} K_{3,1} \\
I_{D}=\rho|\mathbf{y}|^{2} e_{j}^{\perp}\left(-\ell^{2} K_{3,1}+3 \ell^{2}\left(p^{2}+\epsilon^{2}\right) K_{5,1}+|\mathbf{y}|^{2} K_{3,3}-3|\mathbf{y}|^{2}\left(p^{2}+\epsilon^{2}\right) K_{5,3}\right) \\
-3 \rho|\mathbf{y}| p e_{j}\left(\ell^{2}\left[\frac{1}{3} K_{3,0}-\frac{1}{3 R^{3}}\right]-|\mathbf{y}|^{2}\left[K_{3,2}-\frac{1}{3 R^{3}}\right]\right)+3 \rho|\mathbf{y}|^{2} p t e_{j}^{\perp \perp}\left(\ell^{2} K_{5,1}-|\mathbf{y}|^{2} K_{5,3}\right) \\
I_{R}=-2 \rho|\mathbf{y}|^{2} e_{j}^{\perp}\left(2 K_{1,1}-\frac{1}{R}\right)-2 \rho|\mathbf{y}|^{3} p e_{j} K_{3,2}
\end{gathered}
$$

Using the fourth identity, we have $\left(c=q+|\mathbf{y}|, A^{2}=R^{2}+(q+|\mathbf{y}|)^{2}-q^{2}\right)$

$$
\begin{gathered}
K_{1,1}=\frac{1}{|\mathbf{y}|^{2}}(R-A)+\frac{c}{|\mathbf{y}|} K_{1,0} \\
K_{3,1}=-\frac{1}{|\mathbf{y}|^{2}}\left(\frac{1}{R}-\frac{1}{A}\right)+\frac{c}{|\mathbf{y}|} K_{3,0} \\
K_{5,1}=-\frac{1}{3|\mathbf{y}|^{2}}\left(\frac{1}{R^{3}}-\frac{1}{A^{3}}\right)+\frac{c}{|\mathbf{y}|} K_{5,0} \\
K_{3,2}=\frac{1}{|\mathbf{y}|^{2}} K_{1,0}+\frac{c^{2}}{|\mathbf{y}|^{2}} K_{3,0}-\frac{(c+|\mathbf{y}|)}{|\mathbf{y}|^{3}} \frac{1}{R}+\frac{c}{|\mathbf{y}|^{3}} \frac{1}{A} \\
K_{3,3}=\frac{3 c}{|\mathbf{y}|^{3}} K_{1,0}+\frac{c^{3}}{|\mathbf{y}|^{3}} K_{3,0}+\frac{2}{|\mathbf{y}|^{4}}(R-A)-\frac{\left(c^{2}+c|\mathbf{y}|+|\mathbf{y}|^{2}\right)}{|\mathbf{y}|^{4}} \frac{1}{R}+\frac{c^{2}}{|\mathbf{y}|^{4}} \frac{1}{A} \\
K_{5,3}=\frac{2}{|\mathbf{y}|^{3}} K_{5,0}+\frac{c}{|\mathbf{y}|^{3}} K_{3,0}-\frac{1}{3|\mathbf{y}|^{4}}\left(\frac{1}{R}-\frac{1}{A}\right)-\frac{\left(c^{2}+c|\mathbf{y}|+|\mathbf{y}|^{2}\right)}{3|\mathbf{y}|^{4}} \frac{1}{R^{3}}+\frac{c^{2}}{3|\mathbf{y}|^{4}} \frac{1}{A^{3}} \\
K_{5,2}=\frac{1}{3|\mathbf{y}|^{2}} K_{3,0}+\frac{c^{2}}{|\mathbf{y}|^{2}} K_{5,0}-\frac{(c+|\mathbf{y}|)}{3|\mathbf{y}|^{3}} \frac{1}{R^{3}}+\frac{c}{3|\mathbf{y}|^{3}} \frac{1}{A^{3}}
\end{gathered}
$$

so

$$
\begin{aligned}
I_{S} & =\rho e_{j}^{\perp}\left(\left[R-A+c|\mathbf{y}| K_{1,0}\right]+\left(p^{2}+\epsilon^{2}\right)\left[\frac{1}{A}-\frac{1}{R}+c|\mathbf{y}| K_{3,0}\right]\right) \\
& -\rho|\mathbf{y}| p e_{j}\left[K_{1,0}-\frac{1}{R}\right]+\rho|\mathbf{y}|^{2} p t e_{j}^{\perp \perp}\left[\frac{c}{|\mathbf{y}|} K_{3,0}-\frac{1}{|\mathbf{y}|^{2}}\left(\frac{1}{R}-\frac{1}{A}\right)\right]
\end{aligned}
$$




$$
\begin{aligned}
& I_{D}=\rho e_{j}^{\perp}\left(-\ell^{2}\left[\frac{1}{A}-\frac{1}{R}+|\mathbf{y}| c K_{3,0}\right]+\ell^{2}\left(p^{2}+\epsilon^{2}\right)\left[\frac{1}{A^{3}}-\frac{1}{R^{3}}+3 c|\mathbf{y}| K_{5,0}\right]\right. \\
&+ {\left[3 c|\mathbf{y}| K_{1,0}+c^{3}|\mathbf{y}| K_{3,0}+2(R-A)-\left(c^{2}+c|\mathbf{y}|+|\mathbf{y}|^{2}\right) \frac{1}{R}+c^{2} \frac{1}{A}\right] } \\
&-\left.\left(p^{2}+\epsilon^{2}\right)\left[3 c^{3}|\mathbf{y}| K_{5,0}+3 c|\mathbf{y}| K_{3,0}-2\left(\frac{1}{R}-\frac{1}{A}\right)-\left(c^{2}+c|\mathbf{y}|+|\mathbf{y}|^{2}\right) \frac{1}{R^{3}}+c^{2} \frac{1}{A^{3}}\right]\right) \\
&- 3 \rho|\mathbf{y}| p e_{j}\left(\ell^{2}\left[\frac{1}{3} K_{3,0}-\frac{1}{3 R^{3}}\right]-\left[K_{1,0}+c^{2} K_{3,0}-\frac{(c+|\mathbf{y}|)}{|\mathbf{y}|} \frac{1}{R}+\frac{c}{|\mathbf{y}|} \frac{1}{A}-\frac{|\mathbf{y}|^{2}}{3 R^{3}}\right]\right) \\
&+ 3 \rho|\mathbf{y}|^{2} p t e_{j}^{\perp \perp}\left(\ell^{2}\left[-\frac{1}{3|\mathbf{y}|^{2}}\left(\frac{1}{R^{3}}-\frac{1}{A^{3}}\right)+\frac{c}{|\mathbf{y}|} K_{5,0}\right]\right. \\
&-\left.|\mathbf{y}|^{2}\left[\frac{c^{3}}{|\mathbf{y}|^{3}} K_{5,0}+\frac{c}{|\mathbf{y}|^{3}} K_{3,0}-\frac{2}{3|\mathbf{y}|^{4}}\left(\frac{1}{R}-\frac{1}{A}\right)-\frac{\left(c^{2}+c|\mathbf{y}|+|\mathbf{y}|^{2}\right)}{3|\mathbf{y}|^{4}} \frac{1}{R^{3}}+\frac{c^{2}}{3|\mathbf{y}|^{4}} \frac{1}{A^{3}}\right]\right) \\
& I_{R}=-2 \rho e_{j}^{\perp}\left(2\left[(R-A)+c|\mathbf{y}| K_{1,0}\right]-\frac{|\mathbf{y}|^{2}}{R}\right) \\
&-\quad 2 \rho|\mathbf{y}| p e_{j}\left(\left[K_{1,0}+c^{2} K_{3,0}-\frac{(c+|\mathbf{y}|)}{|\mathbf{y}|} \frac{1}{R}+\frac{c}{|\mathbf{y}|} \frac{1}{A}\right]\right)
\end{aligned}
$$

Note that the coefficients of $K_{1,0}$ are

$$
e_{j}^{\perp}|\mathbf{y}| c[\rho+3 \rho-4 \rho]+e_{j} p|\mathbf{y}|[-\rho+3 \rho-2 \rho]
$$

and since $K_{1,0}$ contains a $\log (R)$, the coefficient must be set to zero. This gives $\rho_{1}=2 \rho, \rho=\rho$ and $\rho=\rho$. Then $I_{S}+I_{D}+I_{R}$ at a general point $\mathrm{x}$ is

$$
\begin{gathered}
\rho e_{j}^{\perp}\left[A-R+\left(c^{2}-p^{2}-\epsilon^{2}-\ell^{2}\right) \frac{1}{A}-\left(c^{2}+c|\mathbf{y}|-p^{2}-\epsilon^{2}-\ell^{2}-|\mathbf{y}|^{2}\right) \frac{1}{R}\right. \\
+\quad\left(p^{2}+\epsilon^{2}\right)\left(\ell^{2}-c^{2}\right) \frac{1}{A^{3}}-\left(p^{2}+\epsilon^{2}\right)\left(\ell^{2}-c^{2}-c|\mathbf{y}|-|\mathbf{y}|^{2}\right) \frac{1}{R^{3}} \\
\left.+c|\mathbf{y}|\left(c^{2}-2 p^{2}-2 \epsilon^{2}-\ell^{2}\right) K_{3,0}+3 c|\mathbf{y}|\left(p^{2}+\epsilon^{2}\right)\left(\ell^{2}-c^{2}\right) K_{5,0}\right] \\
+\rho p e_{j}\left[\frac{|\mathbf{y}|\left(\ell^{2}-|\mathbf{y}|^{2}\right)}{R^{3}}+|\mathbf{y}|\left(c^{2}-\ell^{2}\right) K_{3,0}-\frac{c}{R}+\frac{c}{A}\right] \\
+\rho p t e_{j}^{\perp \perp}\left[3 c|\mathbf{y}|\left(\ell^{2}-c^{2}\right) K_{5,0}-2 c|\mathbf{y}| K_{3,0}+\frac{1}{R}-\frac{1}{A}+\left(c^{2}+c|\mathbf{y}|+|\mathbf{y}|^{2}-\ell^{2}\right) \frac{1}{R^{3}}+\left(\ell^{2}-c^{2}\right) \frac{1}{A^{3}}\right]
\end{gathered}
$$

On the sphere surface, this simplifies to

$$
\begin{aligned}
& \rho e_{j}^{\perp}\left[-\frac{R}{2}+\left(a^{2}+\epsilon^{2}+|\mathbf{y}|^{2}\right) \frac{1}{R}-\frac{1}{2}\left(\left(a^{2}+\epsilon^{2}-|\mathbf{y}|^{2}\right)^{2}+4 t^{2}|\mathbf{y}|^{2}\right) \frac{1}{R^{3}}\right] \\
+ & \rho|\mathbf{y}| p e_{j}\left[-\frac{1}{R}+\left(a^{2}+\epsilon^{2}-|\mathbf{y}|^{2}\right) \frac{1}{R^{3}}\right]+2 \rho p t|\mathbf{y}|^{2} e_{j}^{\perp \perp} \frac{1}{R^{3}}
\end{aligned}
$$

after using $\ell^{2}=a^{2}+\epsilon^{2}, A^{2}=R^{2}+c^{2}-q^{2}, p^{2}=R^{2}-q^{2}-t^{2}-\epsilon^{2}, q=c-|\mathbf{y}|, c=|\mathbf{y}|+\alpha-R^{2} \beta$, $\alpha=\left(a^{2}+\epsilon^{2}-|\mathbf{y}|^{2}\right) /(2|\mathbf{y}|)$ and $\beta=1 /(2|\mathbf{y}|)$, and

$$
\begin{gathered}
K_{3,0}=\frac{q+|\mathbf{y}|}{\left(p^{2}+t^{2}+\epsilon^{2}\right)|\mathbf{y}| A}-\frac{q}{\left(p^{2}+t^{2}+\epsilon^{2}\right)|\mathbf{y}| R} \\
K_{5,0}=\frac{(q+|\mathbf{y}|)\left(3 p^{2}+3 t^{2}+3 \epsilon^{2}+2(q+|\mathbf{y}|)^{2}\right)}{3\left(p^{2}+t^{2}+\epsilon^{2}\right)^{2}|\mathbf{y}| A^{3}}-\frac{q\left(3 p^{2}+3 t^{2}+3 \epsilon^{2}+2 q^{2}\right)}{3\left(p^{2}+t^{2}+\epsilon^{2}\right)^{2}|\mathbf{y}| R^{3}}
\end{gathered}
$$




\section{Appendix B. Velocities due to Translation and Rotation of a Sphere}

Consider s sphere of radius $a$ centered at the origin. A uniform translation with velocity $\mathbf{U}$ can be achieved by placing a Stokeslet and a dipole at the center of the sphere, where the dipole strength is proportional to the Stokeslet strength

$$
\mathbf{u}(\mathbf{x})=\mathbf{f}_{0} H_{1}(r)+\left(\mathbf{f}_{0} \cdot \mathbf{x}\right) \mathbf{x} H_{2}(r)+m \mathbf{f}_{0} D_{1}(r)+m\left(\mathbf{f}_{0} \cdot \mathbf{x}\right) \mathbf{x} D_{2}(r) .
$$

The regularizing functions are [19]

$$
H_{1}(r)=\frac{r^{2}+2 \epsilon^{2}}{8 \pi\left(r^{2}+\epsilon^{2}\right)^{3 / 2}}, H_{2}(r)=\frac{1}{8 \pi\left(r^{2}+\epsilon^{2}\right)^{3 / 2}}, D_{1}(r)=\frac{-r^{2}+2 \epsilon^{2}}{8 \pi\left(r^{2}+\epsilon^{2}\right)^{5 / 2}}, D_{2}(r)=\frac{3}{8 \pi\left(r^{2}+\epsilon^{2}\right)^{5 / 2}} .
$$

When $\mathbf{x}$ is on the surface of the sphere, we get

$$
\mathbf{U}=\mathbf{f}_{0}\left(H_{1}(a)+m D_{1}(a)\right)+\left(H_{2}(a)+m D_{2}(a)\right)\left(\mathbf{f}_{0} \cdot \mathbf{x}\right) \mathbf{x}
$$

so that to cancel the last term we choose $m=-H_{2}(a) / D_{2}(a)=\left(a^{2}+\epsilon^{2}\right) / 3$, and

$$
\mathbf{f}_{0}=\left(\frac{D_{2}(a)}{H_{1}(a) D_{2}(a)-H_{2}(a) D_{1}(a)}\right) \mathbf{U}=6 \pi \sqrt{a^{2}+\epsilon^{2}} \mathbf{U}
$$

so that the velocity at any point outside the sphere is

$$
\mathbf{U}_{\text {trans }}(\mathbf{x})=\left(\frac{H_{1}(r) D_{2}(a)-H_{2}(a) D_{1}(r)}{H_{1}(a) D_{2}(a)-H_{2}(a) D_{1}(a)}\right) \mathbf{U}+(\mathbf{U} \cdot \mathbf{x}) \mathbf{x}\left(\frac{H_{2}(r) D_{2}(a)-H_{2}(a) D_{2}(r)}{H_{1}(a) D_{2}(a)-H_{2}(a) D_{1}(a)}\right) .
$$

An uniform rotational velocity $\boldsymbol{\Omega}$ can be achieved by placing a rotlet at the center of the sphere

$$
\mathbf{u}(\mathbf{x})=\mathbf{L} \times \nabla G=(\mathbf{L} \times \mathbf{x}) \frac{G^{\prime}(r)}{r} \quad \text { with } \quad G(r)=-\frac{1}{4 \pi\left(r^{2}+\epsilon^{2}\right)^{1 / 2}},
$$

where $G$ is the regularized Green's function. We define the rotlet strength

$$
\mathbf{L}=a \boldsymbol{\Omega} / G^{\prime}(a)=4 \pi\left(a^{2}+\epsilon^{2}\right)^{3 / 2} \boldsymbol{\Omega}
$$

so that the rotational velocity due to the rotlet is

$$
\mathbf{U}_{\text {rot }}(\mathbf{x})=(\boldsymbol{\Omega} \times \mathbf{x}) \frac{a G^{\prime}(r)}{r G^{\prime}(a)} .
$$

which reduces to $\mathbf{u}(\mathbf{x})=\boldsymbol{\Omega} \times \mathbf{x}$ when $\mathbf{x}$ is on the surface. Velocities due to translation and rotation of a solid sphere given in Eq. (B.2) and Eq. (B.4) are written in terms of regularized functions, but the result is consistent with that for singular elements in [7], when $\epsilon \rightarrow 0$. The formula in Eq. (B.1) gives the regularized version of the Stokes drag law for a solid sphere translated with velocity $\mathbf{U}$ and in the limit of $\epsilon \rightarrow 0$ it approaches the well known quantity $6 \pi \mu a \mathbf{U}$. Similarly, the moment exerted by a rotlet of strength $\mathbf{L}$ on the surface of enclosing sphere is $2 \mathbf{L}$ (equations (8)-(9) in [41]) and for regularization parameter $\epsilon \rightarrow 0$ that leads to the well known torque on the sphere $8 \pi \mu a^{3} \boldsymbol{\Omega}$. 


\section{Appendix C. The rotation of the refrences frame}

The rotation about the centerline of a point $\mathbf{X}_{h}$ on the tube surface is in the direction of

$$
\hat{\theta}=\frac{\mathbf{X}_{h}-\mathbf{X}_{c}}{r_{h}} \times \mathbf{T}=(\mathbf{N} \sin \theta+\mathbf{B} \cos \theta) \times \mathbf{T}=(\mathbf{N} \times \mathbf{T}) \sin \theta+(\mathbf{B} \times \mathbf{T}) \cos \theta
$$

Recall that $\mathbf{N}=\mathbf{B} \times \mathbf{T}$. Then $\mathbf{N} \times \mathbf{T}=-\mathbf{B}$ so that

$$
\hat{\theta}=-\mathbf{B} \sin \theta+\mathbf{N} \cos \theta
$$

To determine the rotation of the coordinates about the centerline, we compute the projection of the last term in velocity onto $\hat{\theta}$ :

$$
r_{h}(\dot{\mathbf{N}} \sin \theta+\dot{\mathbf{B}} \cos \theta) \cdot \hat{\theta}
$$

which should be independent of $\theta$. Expanding, we get

$$
\begin{aligned}
& =r_{h}(\dot{\mathbf{N}} \sin \theta+\dot{\mathbf{B}} \cos \theta) \cdot(-\mathbf{B} \sin \theta+\mathbf{N} \cos \theta) \\
& =r_{h}\left[(\dot{\mathbf{B}} \cdot \mathbf{N}) \cos ^{2} \theta-(\dot{\mathbf{N}} \cdot \mathbf{B}) \sin ^{2} \theta+(\dot{\mathbf{N}} \cdot \mathbf{N}-\dot{\mathbf{B}} \cdot \mathbf{B}) \sin \theta \cos \theta\right]
\end{aligned}
$$

For unit vectors it is the case that $\dot{\mathbf{N}} \cdot \mathbf{N}=\dot{\mathbf{B}} \cdot \mathbf{B}=0$, so we have the rotation

$$
\begin{aligned}
& r_{h}\left[(\dot{\mathbf{B}} \cdot \mathbf{N}) \cos ^{2} \theta-(\dot{\mathbf{N}} \cdot \mathbf{B}) \sin ^{2} \theta\right] \\
= & r_{h}\left[(\dot{\mathbf{B}} \cdot \mathbf{N})-(\dot{\mathbf{B}} \cdot \mathbf{N}+\dot{\mathbf{N}} \cdot \mathbf{B}) \sin ^{2} \theta\right]
\end{aligned}
$$

One can prove that $\dot{\mathbf{B}} \cdot \mathbf{N}+\dot{\mathbf{N}} \cdot \mathbf{B}=0$ as follows: Let $\dot{\mathbf{N}}=N_{t} \mathbf{T}+N_{b} \mathbf{B}$ and $\dot{\mathbf{B}}=B_{t} \mathbf{T}+B_{n} \mathbf{N}$. Since $\mathbf{B}=\mathbf{T} \times \mathbf{N}$ and $\mathbf{T}=\mathbf{N} \times \mathbf{B}$, we have that $\mathbf{B}=(\mathbf{N} \times \mathbf{B}) \times \mathbf{N}$. Differentiating

$$
\begin{aligned}
\dot{\mathbf{B}} & =(\dot{\mathbf{N}} \times \mathbf{B}) \times \mathbf{N}+(\mathbf{N} \times \dot{\mathbf{B}}) \times \mathbf{N}+(\mathbf{N} \times \mathbf{B}) \times \dot{\mathbf{N}} \\
& =\left(\left[N_{t} \mathbf{T}+N_{b} \mathbf{B}\right] \times \mathbf{B}\right) \times \mathbf{N}+\left(\mathbf{N} \times\left[B_{t} \mathbf{T}+B_{n} \mathbf{N}\right]\right) \times \mathbf{N}+\mathbf{T} \times\left[N_{t} \mathbf{T}+N_{b} \mathbf{B}\right] \\
& =N_{t}(\mathbf{T} \times \mathbf{B}) \times \mathbf{N}+B_{t}(\mathbf{N} \times \mathbf{T}) \times \mathbf{N}+N_{b}(\mathbf{T} \times \mathbf{B}) \\
& =0+B_{t} \mathbf{T}-N_{b} \mathbf{N}
\end{aligned}
$$

which shows that $B_{n}=-N_{b}$, or $\dot{\mathbf{B}} \cdot \mathbf{N}=-\dot{\mathbf{N}} \cdot \mathbf{B}$. Therefore, we conclude that the rotation about the centerline is simply

$$
r_{h}(\dot{\mathbf{B}} \cdot \mathbf{N})
$$

which is nonzero for any coordinate system.

\section{References}

[1] L. Fauci, R. Dillon, Biofluidmechanics of reproduction, Annu. Rev. Fluid. Mech. 38 (2006) 371-394.

[2] J. Riffell, R. Zimmer, Sex and flow: the consequences of fluid shear for sperm-egg interactions, J. Exp. Biol. 210 (2007) 3644-3660. 
[3] E. Lauga, T. Powers, The hydrodynamics of swimming microorganisms., Rep. Prog. Phys. 72 (2009) 096601.

[4] M. Short, C. Solari, S. Ganguly, T. Powers, J. Kessler, R. Goldstein, Flows driven by flagella of multicellular organisms enhance long-range molecular transport., Proc. Natl. Acad. Sci. 103 (22) (2006) 8315-8319.

[5] J. R. Blake, A note on the the image system for a Stokeslet in a no-slip boundary, Math. Proc. Cambridge 70 (1971) 303-310.

[6] J. R. Blake, A. T. Chwang, Fundamental singularities of viscous flow, J. Eng. Math. 8 (1) (1974) $23-29$.

[7] C. Pozrikidis, Boundary integral and singularity methods for linearized viscous flow, Cambridge texts in applied mathematics, Cambridge University Press, 1992.

[8] C. W. Oseen, Neuere Methoden und Ergebnisse in der Hydrodynamik, Akad. Verl.-Ges., Leipzig, 1927.

[9] S. D. Nigam, V. Srinivasan, No-slip images in a sphere, J. Math. Phys. Sci. 9 (4) (1975) 389-398.

[10] J. J. L. Higdon, The generation of feeding currents by flagellar motions, J. Fluid. Mech. 94 (1979) 305-330.

[11] S. Kim, S. J. Karrila, Microhydrodynamics: Principles and Selected Applications, Dover Publications, 1991.

[12] C. Maul, S. Kim, Image of a point force in a spherical container and its connection to the Lorentz reflection formula, J. Eng. Math. 30 (1996) 119-130.

[13] N. J. De Mestre, D. F. Katz, Stokes flow about a sphere attached to a slender body, J. Fluid Mech. 64 (1974) 817-826.

[14] J. J. L. Higdon, A hydrodynamic analysis of flagellar propulsion, J. Fluid. Mech. 90 (04) (1979) 685-711.

[15] J. J. L. Higdon, The hydrodynamics of flagellar propulsion: helical waves, J. Fluid. Mech. 94 (1979) 331-351.

[16] S. E. Spagnolie, G. R. Moreno-Flores, D. Bartolo, E. Lauga, Geometric capture and escape of a microswimmer colliding with an obstacle, Soft Matter 11 (2015) 3396-3411.

[17] R. Cortez, The method of regularized Stokeslets, SIAM J. Sci. Comput. 23 (4) (2001) 1204-1225.

[18] R. Cortez, L. Fauci, A. Medovikov, The method of regularized Stokeslets in three dimensions: Analysis, validation, and application to helical swimming, Phys. Fluids 17 (3) (2005) 031504.

[19] J. Ainley, S. Durkin, R. Embid, P. Boindala, R. Cortez, The method of images for regularized Stokeslets, J. Comput. Phys. 227 (9) (2008) 4600-4616.

[20] R. Cortez, D. Varela, A general system of images for regularized Stokeslets and other elements near a plane wall, J. Comput. Phys. 285 (2015) 41-54. 
[21] D. J. Smith, A boundary element regularized stokeslet method applied to cilia- and flagella-driven flow, Proc. R. Soc. A 465 (2009) 3605-3626.

[22] L. Cisneros, J. Kessler, R. Ortiz, R. Cortez, M. Bees, Unexpected bipolar flagellar arrangements and long-range flows driven by bacteria near solid boundaries, Phys. Rev. Lett. 101 (16) (2008) 168102.

[23] J. Gutierrez, M. Sorenson, E. Strawbridge, Modeling fluid flow induced by c. elegans swimming at low reynolds number, in: A.-H. Dediu, M. Lozano, C. Martn-Vide (Eds.), Theory and Practice of Natural Computing, Vol. 8890 of Lecture Notes in Computer Science, Springer International Publishing, 2014, pp. $71-82$.

[24] A. M. Lee, M. A. Berny-Lang, S. Liao, E. Kanso, P. Kuhn, O. J. T. McCarty, P. K. Newton, A lowdimensional deformation model for cancer cells in flow, Phys. Fluids 24 (8) (2012) 081903.

[25] L. Cisneros, R. Cortez, C. Dombrowski, R. Goldstein, J. Kessler, Fluid dynamics of self-propelled organisms, from individuals to concentrated population, Exp. Fluids 43 (2007) 737-753.

[26] E. A. Gillies, R. M. Cannon, R. B. Green, A. A. Pacey, Hydrodynamic propulsion of human sperm, J. Fluid Mech. 625 (2009) 445-474.

[27] J. G. Gibbs, S. Kothari, D. Saintillan, Y. P. Zhao, Geometrically designing the kinematic behavior of catalytic nanomotors, Nano Lett. 11 (6) (2011) 2543-2550.

[28] D. J. Smith, A. A. Smith, J. R. Blake, Mathematical embryology: the fluid mechanics of nodal cilia, J. Eng. Math. 70 (1-3) (2011) 255-279.

[29] J. Simons, S. Olson, R. Cortez, L. Fauci, The dynamics of sperm detachment from epithelium in a coupled fluid-biochemical model of hyperactivated motility, J. Theor. Biol. 354 (0) (2014) 81-94.

[30] B. S. Schuster, J. S. Suk, G. F. Woodworth, J. Hanes, Nanoparticle diffusion in respiratory mucus from humans without lung disease, Biomaterials 34 (13) (2013) 3439-3446.

[31] P. Kozlovsky, A. Gefen, The relative contributions of propulsive forces and receptor-ligand binding forces during early contact between spermatozoa and zona pellucida of oocytes, J. Theor. Biol. 294 (2012) 139143.

[32] J. K. Wróbel, R. Cortez, L. Fauci, Modeling viscoelastic networks in Stokes flow, Phys. Fluids 26 (2014) 113102.

[33] T. Fenchel, How dinoflagellates swim, Protist 152 (2001) 329-338.

[34] I. Miyasaka, K. Nanba, K. Furuya, Y. Nimura, A. Azuma, Functional roles of the transverse and longitudinal flagella in the swimming motility of Prorocentrum minimum (Dinophyceae)., J. Exp. Biol. 207 (2004) 30553066 . 
[35] H. Nguyen, R. Cortez, L. Fauci, Computing flows around microorganisms: slender-body theory and beyond, Amer. Math Monthly 121 (9) (2014) 810-823.

[36] H. Nguyen, R. Ortiz, R. Cortez, L. Fauci, The action of waving cylindrical rings in a viscous fluid, J. Fluid Mech. 671 (2011) 574-586.

[37] J. Martingale, M. Jabbarzadeh, H. Fu, Choice of computational method for swimming and pumping with helical filaments at low reynolds number, Phys. Fluids 28 (2016) 021901.

[38] J. G. Brasseur, M. Nicosia, A. Pal, M. L., Function of longitudinal vs circular muscle fibers in esophageal peristalsis, deduced with mathematical modeling, World J. Gastroentero. 13 (2007) 1335-1346.

[39] J. Jimenez-Lozano, M. Sen, P. Dunn, Particle motion in unsteady two-dimensional peristaltic flow with application to the ureter, Phys. Rev. E 79 (2009) 041901.

[40] S. Yaniv, A. Jaffa, D. Elad, Modeling embryo transfer in a closed uterine cavity, J. Biomech. Engr. 134 (2012) 111003-1.

[41] A. T. Chwang, T. Y.-T. Wu, Hydromechanics of low-Reynolds-number flow. part 2. singularity method for stokes flows, Journal of Fluid Mechanics 67 (1975) 787-815. 